\section{JOURNAL OF Economic Theory}

www.elsevier.com/locate/jet

\title{
A solution to the two-person implementation problem
}

\author{
Jean-François Laslier ${ }^{a}$, Matías Núñez ${ }^{b, *}$, M. Remzi Sanver ${ }^{c}$ \\ ${ }^{\text {a }}$ CNRS, Paris School of Economics, France \\ ${ }^{\mathrm{b}}$ CREST, CNRS, Ecole Polytechnique, GENES, ENSAE Paris, Institut Polytechnique de Paris, 91120 Palaiseau, France \\ ${ }^{\mathrm{c}}$ Université Paris-Dauphine, Université PSL, CNRS, LAMSADE, 75016 Paris, France
}

Received 9 July 2020; final version received 19 March 2021; accepted 19 April 2021

Available online 26 April 2021

\begin{abstract}
We propose strike mechanisms as a solution to the classical problem of Hurwicz and Schmeidler (1978) and Maskin (1999) according to which, in two-person societies, no Pareto efficient rule is Nashimplementable. A strike mechanism specifies the number of alternatives that each player vetoes. Each player simultaneously casts these vetoes and the mechanism selects randomly one alternative among the non-vetoed ones. For strict preferences over alternatives and under a very weak condition for extending preferences over lotteries, these mechanisms are deterministic-in-equilibrium. They Nash implement a class of Pareto efficient social choice rules called Pareto-and-veto rules. Moreover, under mild richness conditions on the domain of preferences over lotteries, any Pareto efficient Nash-implementable rule is a Pareto-and-veto rule and hence is implementable through a strike mechanism.
\end{abstract}

(C) 2021 Elsevier Inc. All rights reserved.

JEL classification: D71; D72

Keywords: Nash implementation; Two players; Pareto efficiency

\footnotetext{
We would like to thank Lorenzo Bastianello, Felix Brandt, Chris Chambers, Bhaskar Dutta, Margarita Kirneva, Yukio Koriyama, Matthew Jackson, Margaret Meyer, Phil Reny, Larry Samuelson and Arunava Sen for useful remarks as well as an anonymous referee and the associate editor. We are particular indebted to William Thomson for his comments and suggestions. Financial support from the project IDEX ANR-10-IDEX-0001-02 PSL MIFID and from the project ANR-11-IDEX-0003/Labex Ecodec/ANR-11-LABX-0047 is gratefully acknowledged. Jean-François Laslier thanks the support of the EUR grant ANR-17-EURE-0001.

* Corresponding author.

E-mail address: matias.nunez@ensae.fr (M. Núñez).
} 


\section{Introduction}

Can one design some protocol that ensures that two players reach a Pareto efficient agreement in equilibrium? The theorems of Hurwicz and Schmeidler (1978) and Maskin (1999), at the outset of implementation theory, provide a negative answer to this question: no deterministic mechanism, except dictatorship, can guarantee that every Nash equilibrium is Pareto efficient. In fact, there is a tension between the conditions for the existence of an equilibrium at every preference profile and those which ensure that each outcome is Pareto efficient. We refer to this impossibility as the two-person implementation problem.

We propose a solution to this problem based on "strike" mechanisms. ${ }^{1}$ A strike mechanism endows each player $i$ with $v_{i}$ vetoes to be distributed among the alternatives, with $v_{1}+v_{2}$ being equal to the number of alternatives minus one, so that at least one alternative remains non-vetoed. The game is simultaneous and the outcome is the uniform lottery over the non-vetoed alternatives.

By allowing lotteries, we introduce a modification of the mechanisms used in general for implementation but, as we shall prove, lotteries do not materialize at equilibrium; they only act as off-equilibrium threats. From a mechanism design perspective, we therefore consider Nash implementation through deterministic-in-equilibrium mechanisms or simply DE mechanisms.

The idea of introducing off-equilibrium threats is already present in the implementation literature. ${ }^{2}$ More precisely, Sanver (2006) allows for off-equilibrium awards, Bochet (2007) considers lotteries whereas Benoît and Ok (2008) consider mechanisms with awards and mechanisms with lotteries off-equilibrium. But these papers consider three players or more while we consider the two-person case. This aspect is important since the characterizations of Moore and Repullo (1990) and Dutta and Sen (1991) jointly with the mentioned impossibility results suggest that, with two players, "exact implementation is very demanding, at least in the absence of domain restrictions" as Abreu and Sen (1991) puts it, whereas Nash-implementable rules of interest exist with three or more players. Another difference is that our work, rather than relying on integer games, ${ }^{3}$ builds games -the strike mechanisms- whose rules are simple and explicitly based on vetoes.

Since we deal with lotteries, the notion of Pareto efficiency needs some qualification (see Bogomolnaia and Moulin (2001) for a discussion). Two classical definitions are ex-ante and ex-post Pareto efficiency. A lottery is ex-ante Pareto efficient if no other lottery Pareto dominates it, whereas it is ex-post Pareto efficient if no alternative that can be selected by the lottery is Pareto dominated by some other alternative. While we show that the possibility of ex-ante Pareto efficient implementation cannot be hoped for, we establish that ex-post Pareto efficient implementation is possible, by DE mechanisms, as soon as preferences over alternatives are strict. ${ }^{4}$

Our main result is that a SCR is Pareto efficient and Nash-implementable by a DE mechanism if and only if it is a Pareto-and-veto rule: for some pair of integers $v=\left(v_{1}, v_{2}\right)$, with $v_{1}+v_{2}+1$

\footnotetext{
1 The word "strike" comes from legal American vocabulary. See for instance the rules of the American Arbitration Association for arbitrator selection: https://www.adr.org/ArbitratorSelection.

2 Randomization off-equilibrium is used in other branches of economic theory. See for instance Ederer et al. (2018) for recent work in the theory of incentives where similar techniques are used as a strategy to combat gaming by better informed agents.

3 Jackson (2001) summarizes some views on the limits of these games.

4 The current results do not extend to the setting where the players are indifferent among several alternatives. Indeed, as proved by Sanver (2006), no selection of the Pareto set is (Maskin) monotonic and hence can be implemented.
} 
being the number of alternatives, it selects all Pareto efficient alternatives that are not among the $v_{i}$ worst alternatives for each player $i$. The Pareto-and-veto rule with vector $v$ is denoted $\mathrm{PV}_{v}{ }^{5}$ We show that the strike mechanism with vetoes $v_{1}$ and $v_{2}$ Nash implements $\mathrm{PV}_{v}$.

The study of the strike mechanism is facilitated by the fact that the best-response reasoning is straightforward in this game. Given the vetoes cast by her opponent, a player can induce any non-vetoed alternative as the outcome by adequately casting her vetoes. Thus, her best response amounts to select her best element among the non-vetoed alternatives.

We prove that, when preferences are strict, the equilibria of this game are pure and strict. Then, the nice feature of best responses has three consequences for equilibrium behavior. First, each veto mechanism is $\mathbf{D E}$, with a unique alternative that remains non-vetoed in equilibrium. Second, any equilibrium outcome is Pareto efficient. Third, the equilibrium strategies have a natural shape: if $x$ is the implemented alternative and $v_{i}$ is the number of vetoes, player $i$ vetoes all alternatives preferred to $x$ by her opponent (say $k$ alternatives) and she vetoes also $v_{i}-k$ among the alternatives less preferred than $x$ by her opponent. If both strategies veto disjoint sets of alternatives, this forces each player to accept her opponent's strategy. In any equilibrium, this is the case: the players veto disjoint sets of alternatives and only one alternative, the implemented one, remains non-vetoed.

All these results hold under the standard von Neumann and Morgenstern expected utility framework but are even more general than that. Indeed, they remain true under a mild condition that we term "best-element bias": for any set of alternatives, a player prefers the sure lottery that consists of her most preferred element in the set to any lottery with support in the same set. As a matter of fact, we could define the output of the mechanism as an irresolute set of alternatives rather than a lottery and obtain equivalent results, under very mild hypotheses to extend preferences over alternatives to sets.

At this point, we have described a solution to the two-person implementation problem: a mechanism that implements a Paretian SCR. We then show that, in some sense, there cannot be a different solution. This necessity part is more involved. Here, the key concept is the veto power generated by a mechanism: a mechanism $\mu$ endows player $i$ with veto power over some set $X$ of alternatives if and only if player $i$ has some strategy that prevents any alternative in $X$ to be selected with positive probability whatever her opponent plays. As we show, any mechanism $\mu$ that ensures Pareto efficient outcomes must endow each player $i$ with veto power over every set of alternatives whose cardinality does not exceed some integer $v_{i}^{\mu}$ with $v_{1}^{\mu}+v_{2}^{\mu}+1$ being the number of alternatives. This is a strong result which almost directly entails that only subcorrespondences of $\mathrm{PV}_{v}$ are Nash-implementable. The necessity is established on a domain of preference extensions over lotteries that is rich enough to include specific extended preferences that we label "priority" extensions. In words, a "priority" extended preference is defined by the property that whenever all the elements of a set $X$ are preferred to all elements outside $X$, any lottery that put some weight (however small) on some element of $X$ is preferred to any lottery that puts no weight on $X$. For instance, the domain of vNM preferences satisfies this requirement.

We also identify a set of conditions that characterize the class of Pareto-and-veto rules which, thus, turn to be necessary and sufficient for two-person Nash implementability with DE mecha-

\footnotetext{
5 It is not the first time that Pareto-and-veto rules are found to be of interest in the literature. Moulin (1983) defines PV under the name "veto core" (Chapter 6.5). Abreu and Sen (1991) (pp. 1016-17) present this class of rules as the main example that is virtually implementable but fails to be Nash-implementable. In a setting where monetary transfers are allowed, Sanver (2018) designs a direct veto mechanism that implements alternatives which are Pareto efficient and preferable to some disagreement outcome by both players.
} 
nisms. As such, our conditions are weaker than the necessary and sufficient conditions identified by Moore and Repullo (1990) and Dutta and Sen (1991) for two-person Nash implementability without DE mechanisms, as their conditions coincided with dictatorship over the full domain of preferences. These conditions are Pareto efficiency, Maskin monotonicity, neutrality-on-itsvetoes (a weakening of neutrality) and the intersection property which is the key distinction between two-player and many-player implementation models. The conditions are independent, as shown in Appendix C.

The structure of the paper is as follows: Section 2 introduces the basic notions and Section 3 presents the strike mechanisms. Section 4 presents the outcomes of these mechanisms and tackles the necessity issue. Section 5 shows how the current results are related to the classical characterization of Nash-implementable social choice rules with two players. Section 6 presents a review of the various other ideas that have emerged in the literature to bypass the Hurwicz-Schmeildler impossibility of Paretian implementation and makes some concluding remarks.

\section{Basic notions and notation}

A set $N=\{1,2\}$ of two players faces a finite set $A$ of $n+1 \geq 3$ alternatives. We write $\mathcal{A}=2^{A}$ for the power set of $A$. The set of probability distributions (or "lotteries") over $A$ is denoted $\Delta=$ $\left\{p: A \rightarrow[0,1] \mid \sum_{x \in A} p(x)=1\right\}$. For each lottery $p \in \Delta$, we let $\operatorname{SUPP}(p)=\{x \in A \mid p(x)>0\}$ denote the support of $p$. For each $X \in \mathcal{A}, p[X]=\sum_{x \in X} p(x)$ stands for the probability that $p$ selects an alternative in $X$. Let $\Delta^{\text {uni }}$ denote the set of all uniform probability distributions over the non-empty subsets of $A$. Slightly abusing notation, we let $\{x\}$ denote both the singleton set consisting of alternative $x$ and the lottery that selects $x$ with probability one.

The set of linear orders over $A$ is denoted by $\mathcal{L}_{A}$ and its generic element $\succ_{i}$ is the preference of $i \in N{ }^{6}$ The set of (strict) preference profiles over $A$ is $\mathcal{L}_{A}^{2}=\mathcal{L}_{A} \times \mathcal{L}_{A}$ with $\succ=\left(\succ_{1}, \succ_{2}\right)$ denoting a generic preference profile. We write

$$
\mathrm{PO}(\succ)=\left\{x \in A \mid \nexists y \in A: \forall i \in N, y \succ_{i} x\right\}
$$

for the set of Pareto optimal alternatives at $\succ \in \mathcal{L}_{A}^{2}$.

Let $L\left(x, \succ_{i}\right)=\left\{y \in A: x \succ_{i} y\right\}$ and $U\left(x, \succ_{i}\right)=\left\{y \in A: y \succ_{i} x\right\}$ respectively denote the (strict) lower contour set and the (strict) upper contour set of $x \in A$ at $\succ_{i} \in \mathcal{L}_{A}$.

A social choice rule (SCR) is a mapping $f: \mathcal{L}_{A}^{2} \rightarrow \mathcal{A} \backslash\{\emptyset\}$. A SCR is Pareto efficient iff $f(\succ) \subseteq \mathrm{PO}(\succ)$ for all $\succ \in \mathcal{L}_{A}^{2}$. We say that $f$ is a sub-correspondence of $g$ and write $f \subseteq g$ whenever $f(\succ) \subseteq g(\succ)$ for all $\succ \in \mathcal{L}_{A}^{2}$.

A mechanism is a function $\mu: \mathcal{M} \rightarrow \Delta$ with $\mathcal{M}=\mathcal{M}_{1} \times \mathcal{M}_{2}$ where $\mathcal{M}_{i} \neq \varnothing$ is the message space of $i \in N$. In order to properly define the game associated to $\mu$, we do not need to extend preferences over the whole $\Delta$ but simply over $\mu(\mathcal{M}):=\{p \in \Delta \mid p=\mu(m)$ for some $m \in \mathcal{M}\}$, the range of $\mu$. We only consider mechanisms with finite ranges. ${ }^{7}$ For example, the set of uniform lotteries over $A$, denoted $\Delta^{\text {uni }}$, is finite. The strike mechanisms, which play the central role in this work, have $\Delta^{\text {uni }}$ as their range.

\footnotetext{
6 More precisely, one of $x \succ_{i} y$ and $y \succ_{i} x$ holds for any distinct $x, y \in A$ while $x \succ_{i} x$ fails for all $x \in A$. Moreover, $x \succ_{i} y$ and $y \succ_{i} z$ implies $x \succ_{i} z$ for all $x, y, z \in A$.

7 While our results still hold extending over the whole simplex, the richness condition PREX becomes harder to satisfy. We would like to thank Bhaskar Dutta for pointing this out.
} 
We let $\mathcal{P}_{\mu(\mathcal{M})}$ stand for the set of complete and transitive binary relations over $\mu(\mathcal{M})$. A typical element of $\mathcal{P}_{\mu(\mathcal{M})}$ is denoted $\succeq_{i}^{*}$ with $\succ_{i}^{*}$ being its strict part. We say that $\succeq_{i}^{*}$ is an extension of $\succ_{i}$ when $x \succ_{i} y \Longrightarrow\{x\} \succeq_{i}^{*}\{y\}, \forall x, y \in A$ with $\{x\},\{y\} \in \mu(\mathcal{M})$.

For a mechanism $\mu: \mathcal{M} \rightarrow \Delta$ and a preference profile over lotteries $\succeq^{*}=\left(\succeq_{1}^{*}, \succeq_{2}^{*}\right)$, a Nash equilibrium is a pair of messages $\left(m_{1}, m_{2}\right) \in \mathcal{M}$ such that, for all $m_{1}^{\prime} \in \mathcal{M}_{1}$ and all $m_{2}^{\prime} \in \mathcal{M}_{2}$, $\mu\left(m_{1}, m_{2}\right) \succeq_{1}^{*} \mu\left(m_{1}^{\prime}, m_{2}\right)$ and $\mu\left(m_{1}, m_{2}\right) \succeq_{2}^{*} \mu\left(m_{1}, m_{2}^{\prime}\right)$. Let $\mathcal{N}^{\mu}\left(\succeq^{*}\right)$ denote the set of Nash equilibria of the mechanism $\mu$ at the preference profile $\succeq^{*}$.

We now turn to the question of the domain of preferences to be considered. As already mentioned we work under the assumption that preferences over alternatives are strict, but we are flexible as to the way preferences are extended from alternatives to lotteries. Since there are many ways to do so, we use a notion of admissible extended preferences. Let $\kappa\left(\succ_{i}\right) \subseteq \mathcal{P}_{\Delta}$ be a set of admissible preferences over lotteries of player $i$ associated with $\succ_{i} \in \mathcal{L}_{A}$. Abusing notation, let $\kappa(\succ) \subseteq \mathcal{P}_{\Delta}^{2}$ be the set of admissible preference profiles over $\Delta$ associated with the preference profile $\succ=\left(\succ_{1}, \succ_{2}\right)$. Such a correspondence $\kappa$ that associates to each preference a set of extended preferences (and to each profile of preference a set of profiles of extended preferences) is called a domain of preference extensions.

Given a domain $\kappa$, a mechanism $\mu$ is admissible iff for all $\succ \in \mathcal{L}_{A}^{2}$ and all $\succeq^{*} \in \kappa(\succ), \mathcal{N}^{\mu}\left(\succeq^{*}\right.$ ) $\neq \varnothing$. It is deterministic-in-equilibrium (DE) iff for all $\succ \in \mathcal{L}_{A}^{2}$, all $\succeq^{*} \in \kappa(\succ)$, and all $m \in$ $\mathcal{N}^{\mu}\left(\succeq^{*}\right)$, \#supp $(\mu(m))=1$. It Nash-implements the SCR $f: \mathcal{L}_{A}^{2} \rightarrow \mathcal{A}$ iff for all $\succ \in \mathcal{L}_{A}^{2}$ and all $\succeq^{*} \in \kappa(\succ), f(\succ)=\bigcup_{m \in \mathcal{N} \mu\left(\succeq^{*}\right)} \operatorname{SUPP}(\mu(m))$. Note that if $\mu$ Nash-implements some SCR $f$, then $\mu$ is admissible.

Throughout the paper we use two properties of preference extensions. The first one, bestelement bias, can be stated as follows: a player with a best-element bias prefers the sure lottery that selects her best element in $X$ to any lottery with support in $X$.

Best-element bias: Let $\succ_{i} \in \mathcal{L}_{A}$ be a preference on $A$, and let $\bar{\Delta} \subseteq \Delta$ be a set of lotteries. An extension $\succeq_{i}^{*}$ of $\succ_{i}$ exhibits the best-element bias in $\bar{\Delta}$ when for any $X \in \mathcal{A}$ with $\# X>1$ and any $x \in X$, if $x \succ_{i} y$ for any $y \in X \backslash\{x\}$, then $\{x\} \succ_{i}^{*} p$ for all $p \in \bar{\Delta}$ with $\operatorname{SupP}(p) \subseteq X$ and $p \neq\{x\}$.

A domain $\kappa$ is said to satisfy the best-element bias (in short: $\kappa$ satisfies BEB) in $\bar{\Delta}$ if, for any strict preference $\succ_{i} \in \mathcal{L}_{A}$, any extension $\succeq_{i}^{*} \in \kappa\left(\succ_{i}\right)$ exhibits the best-element bias in $\bar{\Delta}$. Note that BEB is satisfied by virtually all domains of preference extensions that are considered in the literature, ${ }^{8}$ including the von Neumann and Morgenstern domain.

The second condition, priority extension, deals with the richness of the domain of preference extensions. For any lottery $p \in \Delta$, we write $p\left[U\left(x, \succeq_{i}\right)\right]=\sum_{y: y \succeq_{i} x} p(y)$ to refer to the probability, according to $p$, of obtaining an alternative at least as good as $x$ according to $\succ_{i}$.

Priority extension: Let $\succeq_{i}^{*}$ extend $\succ_{i}$ and let $x \in A$. The extension $\succeq_{i}^{*}$ is a priority extension (PREX) of $\succ_{i}$ for $x$ in $\bar{\Delta}$ iff given any two lotteries $p, q \in \bar{\Delta}$, if $p\left[U\left(x, \succeq_{i}\right)\right]>0$ and $q\left[U\left(x, \succeq_{i}\right.\right.$ )$]=0$, then $p \succ_{i}^{*} q$.

\footnotetext{
8 In fact, BEB is satisfied if one considers the well-known preference extension axioms of the literature (such as Gärdenfors (1976) or Kelly (1977)) and deduces preferences over lotteries through the preferences over their supports. If $\kappa$ satisfies BEB (which is universally quantified), every sub-correspondence of $\kappa$ satisfies $\mathbf{B E B}$ as well.
} 
The interpretation of this property is clear: under a priority extension, some alternative $x$ is used as a grading benchmark: The individual prefers to reach the benchmark $x$, even with a tiny probability, than not reaching it. ${ }^{9}$

While PREX may seem related to lexicographic preferences, we now show by example that PREX is compatible with expected utilities while, as is well known, lexicographic preferences fail to be so. Consider a domain of extension that satisfies the condition in the set $\Delta^{\text {uni }}$ of uniform lotteries. Similar examples can be found for any finite set of lotteries. Consider the correspondence $\kappa^{\mathrm{VNM}}: \mathcal{L}_{A} \rightarrow \mathcal{P}_{\Delta}$ uni that allows any von Neumann and Morgenstern extension of $\succ_{i}$. In other words, for $\succ_{i} \in \mathcal{L}_{A}, \kappa^{\mathrm{vNM}}\left(\succ_{i}\right)$ is the set of all $\succeq_{i}^{*} \in \mathcal{P}_{\Delta^{\text {uni }}}$ such that there exists a vector $u \in \mathbb{R}^{A}$ with $a \succ_{i} b \Longleftrightarrow u_{a}>u_{b}$ for all $a, b \in A$ and:

$$
\forall p, q \in \Delta^{\mathrm{uni}}, p \succ_{i}^{*} q \Longleftrightarrow \sum_{a \in A} p(a) u_{a}>\sum_{a \in A} q(a) u_{a}
$$

The domain $\kappa^{\mathrm{VNM}}(>)$ contains priority extensions of $\succ$ to $\Delta^{\text {uni }}$. To see this, label the alternatives in $A$ according to the preference: $a_{n+1} \succ_{i} a_{n} \succ_{i} \ldots \succ_{i} a_{1}$ and let $u_{a_{k}}=(n+1)^{k}$ for any $a_{k} \in A$. Take any pair $p, q \in \Delta^{\text {uni }}$ with $p\left[U\left(a_{k}, \succeq_{i}\right)\right]>0$ and $q\left[U\left(a_{k}, \succeq_{i}\right)\right]=0$ for some $a_{k}$. The expected value of $p$, that is $\sum_{a \in A} p(a) u_{a}$, reaches its minimum when the lottery contains in its support $a_{k}$ but no better alternative according to $\succeq_{i}$ (and hence has $k$ alternatives in its support). The expected value $\sum_{a \in A} p(a) u_{a}$ is at least

$$
\frac{u_{a_{k}}}{k}>\frac{u_{a_{k}}}{k+1}=\frac{(n+1)^{k}}{k+1} \geq(n+1)^{k-1} .
$$

The expected value of $q, \sum_{a \in A} q(a) u_{a}$, reaches its maximum when $q=\left\{a_{k-1}\right\}$ and hence its value is at most $(n+1)^{k-1}$. Therefore, for any $a_{k} \in A, p\left[U\left(a_{k}, \succeq_{i}\right)\right]>0$ and $q\left[U\left(a_{k}, \succeq_{i}\right)\right]=0$ implies that $p \succ_{i}^{*} q$. Thus, uniform lotteries are ordered following the priority rule.

We say that a domain $\kappa$ satisfies PREX in $\bar{\Delta}$ iff for all $\succ_{i} \in \mathcal{L}_{A}^{2}$ and for all $x \in A$, there is some $\succeq_{i}^{*} \in \kappa\left(\succ_{i}\right)$ that is a priority extension of $\succ_{i}$ in $\bar{\Delta}$ for $x$.

\section{The strike mechanism}

\subsection{Definition}

A strike mechanism endows each player $i \in N$ with a non-negative number $v_{i}$ of vetoes, with $v_{1}+v_{2}=n$. The set $\mathcal{M}_{i}=\left\{X \subseteq A \mid \# X=v_{i}\right\}$ represents the sets of alternatives $i$ can veto, and $\mathcal{M}=\mathcal{M}_{1} \times \mathcal{M}_{2}$ is the joint message space.

Definition 1. The strike mechanism $\mu_{v}: \mathcal{M} \rightarrow \Delta^{\text {uni }}$ associates to each pair of messages $m=$ $\left(m_{1}, m_{2}\right)$, the lottery $\mu_{v}(m)$ that is uniform over the set $\operatorname{SUPP}\left(\mu_{v}(m)\right)=A \backslash\left(m_{1} \cup m_{2}\right)$.

In other words, an alternative is uniformly drawn from the non-vetoed alternatives. Note that, as $v_{1}+v_{2}=n$, the set $m_{1} \cup m_{2}$ contains at most $n$ elements, so that $\operatorname{SUPP}\left(\mu_{v}(m)\right)$ is always non-empty. Our results would remain unaffected under an alternative specification of the strike

\footnotetext{
9 Note that if $x$ is bottom-ranked in $\succ$, there is no lottery $q$ with $q[U(x, \succeq)]=0$, so that any extension is (vacuously) a priority extension for $x$.
} 
mechanism in which the mechanism selects one among the non-vetoed alternatives according to any probability distribution with full support over these alternatives.

In order to study the mechanism $\mu_{v}$, we introduce the following notation. Let

$$
g_{v}\left(\mathcal{M}_{i}, m_{j}\right)=\left\{X \in \mathcal{A} \mid \operatorname{SUPP}\left(\mu_{v}\left(m_{i}, m_{j}\right)\right)=X \text { for some } m_{i} \in \mathcal{M}_{i}\right\}
$$

denote the attainable set for player $i$ at $m_{j}$ under $\mu_{v}$. This set contains the different supports of the uniform lotteries that player $i$ can induce when player $j$ selects $m_{j}$ under the strike mechanism $\mu_{v}$. Because of the number of vetoes at her disposal, player $i$ can choose the support of the outcome by adequately casting her vetoes as described by the following result:

Lemma 1. For each player $i$ and each strategy $m_{j} \in \mathcal{M}_{j}$, the attainable set of the strike mechanism $\mu_{v}$ equals:

$$
g_{v}\left(\mathcal{M}_{i}, m_{j}\right)=\left\{X \subseteq A \backslash m_{j} \mid 1 \leq \# X \leq \min \left\{n+1-v_{i}, n+1-v_{j}\right\}\right\}
$$

Proof. Take some player $i$ and some strategy $m_{j} \in \mathcal{M}_{j}$. Take first the case with $v_{i}<v_{j}$ so that $n+1-v_{j}<n+1-v_{i}$. We want to prove that for each non-empty $X \subseteq A \backslash m_{j}$ (hence with $\left.\# X \leq n+1-v_{j}\right)$, there is some $m_{i} \in \mathcal{M}_{i}$ with $\operatorname{SUPP}\left(\mu_{v}\left(m_{i}, m_{j}\right)\right)=X$. Note that each nonempty subset of $A \backslash m_{j}$ is of the form $A \backslash\left(m_{j} \cup C\right)$ with $0 \leq \# C \leq v_{i}$. Thus, it suffices to pick $m_{i}$ such that $m_{i} \backslash m_{j}=C$ which ensures that $\operatorname{SUPP}\left(\mu_{v}\left(m_{i}, m_{j}\right)\right)=A \backslash\left(m_{i} \cup m_{j}\right)=A \backslash\left(m_{j} \cup C\right)$, as required. In the case $v_{i} \geq v_{j}$, take $m_{i}$ with $m_{i} \backslash m_{j}=C$. Since $v_{i} \geq v_{j}$, it follows that \#C $\geq$ $v_{i}-v_{j}$ and hence for each non-empty $X \subseteq A \backslash m_{j}$ with $\# X \leq n+1-v_{j}-\left(v_{i}-v_{j}\right)=n+1-v_{i}$, there is some $m_{i} \in \mathcal{M}_{i}$ with $\operatorname{SUPP}\left(\mu_{v}\left(m_{i}, m_{j}\right)\right)=X$.

\subsection{Best responses}

Lemma 1's main implication is that player $i$ can induce any singleton in $A \backslash m_{j}$ as the support of the outcome: formally, for any player $i$ and any alternative $x \in A$ :

$$
x \in A \backslash m_{j} \Longrightarrow\{x\} \in g_{v}\left(\mathcal{M}_{i}, m_{j}\right) .
$$

Best responses can thus be easily described, as done in the following statement. For each strategy $m_{j}$ of player $j$, let $x_{i}\left(m_{j}\right)$ be $i$ 's preferred non-vetoed alternative so that $\left\{x_{i}\left(m_{j}\right)\right\}=$ $\arg \max _{X \backslash m_{j}} \succ_{i}$.

Proposition 1. Let the domain $\kappa$ satisfies $\boldsymbol{B E B}$ in the range of $\mu_{v}$. For each strategy $m_{j}$ of her opponent, player $i$ has a unique best response to $m_{j}$, denoted $m_{i}^{*}\left(m_{j}\right)$, with

$$
m_{i}^{*}\left(m_{j}\right)=X \backslash\left(m_{j} \cup\left\{x_{i}\left(m_{j}\right)\right\}\right) \quad \text { and } \quad \mu_{v}\left(m_{i}^{*}\left(m_{j}\right), m_{j}\right)=\left\{x_{i}\left(m_{j}\right)\right\} \text {. }
$$

Proof. The assumption that preferences over alternatives are strict implies that $x_{i}\left(m_{j}\right)$ is welldefined, and the assumption that the preferences over lotteries satisfy BEB implies that $x_{i}\left(m_{j}\right)$ is preferred to any other possible outcome. Lemma 1 indicates that $x_{i}\left(m_{j}\right)$ belongs to the attainable set $g_{v}\left(\mathcal{M}_{i}, m_{j}\right)$ for any $m_{j} \in \mathcal{M}_{j}$. It is thus the unique best possible outcome, and it is obtained by eliminating all other non-vetoed alternatives, as indicated. 
Table 1

The Pareto-and-veto rule $\mathrm{PV}_{(1,1)}$.

\begin{tabular}{lllllll}
\hline & $a b c$ & $a c b$ & $b a c$ & $b c a$ & $c a b$ & $c b a$ \\
\hline$a b c$ & $a$ & $a$ & $\{a, b\}$ & $b$ & $a$ & $b$ \\
$a c b$ & $a$ & $a$ & $a$ & $c$ & $\{a, c\}$ & $c$ \\
$b a c$ & $\{a, b\}$ & $a$ & $b$ & $b$ & $b$ & $b$ \\
$b c a$ & $b$ & $c$ & $b$ & $c$ & $c$ & $\{b, c\}$ \\
$c a b$ & $a$ & $\{a, c\}$ & $a$ & $\{b, c\}$ & $c$ & $c$ \\
$c b a$ & $b$ & $c$ & $b$ & & $c$ \\
\hline
\end{tabular}

\subsection{Equilibrium}

The first consequence of Proposition 1 is that strike mechanisms are deterministic in equilibrium as long as the domain satisfies BEB.

Proposition 2. For any strike mechanism $\mu_{v}$, if the domain $\kappa$ satisfies $\boldsymbol{B E B}$ in the range of $\mu_{v}$, then $\mu_{v}$ is $\boldsymbol{D E}$.

Proof. Assume that there is some equilibrium $m=\left(m_{1}, m_{2}\right)$ with \#SUPP $\left(\mu_{v}(m)\right)>1$. Consider some player $i$ and some alternative $x \in \operatorname{SUPP}\left(\mu_{v}(m)\right)$ with $x \succeq_{i} y$ for all $y \in \operatorname{SUPP}\left(\mu_{v}(m)\right)$. Since $x \in A \backslash m_{j}$, Lemma 1 shows that $\{x\} \in g_{v}\left(\mathcal{M}_{i}, m_{j}\right)$. Thus, there is some $m_{i}^{\prime} \in \mathcal{M}_{i}$ with $\mu_{v}\left(m_{i}^{\prime}, m_{j}\right)=\{x\}$. Furthermore, $\{x\} \succ_{i}^{*} \mu_{v}(m)$ due to BEB, which contradicts that $m$ is an equilibrium.

Since a strike mechanism is $\mathbf{D E}$, no uncertainty remains in equilibrium: players veto disjoint sets of alternatives and a unique alternative is selected.

\section{Pareto efficient implementation}

The previous section has studied the game-theoretical properties of the proposed mechanism. We now study its outcomes.

\subsection{The Pareto-and-veto correspondence $\mathrm{PV}_{v}$}

Definition 2. For any $v=\left(v_{1}, v_{2}\right) \in\{0,1, \ldots, n\}^{2}$ with $v_{1}+v_{2}=n$, the Pareto-and-veto rule $\mathrm{PV}_{v}: \mathcal{L}_{A}^{2} \rightarrow \mathcal{A}$ is the SCR:

$$
\mathrm{PV}_{v}(\succ)=\overbrace{\mathrm{PO}(\succ)}^{\text {Pareto }} \cap \overbrace{\underbrace{\left\{x \in A \mid \# L\left(x, \succ_{1}\right) \geq v_{1}\right\}}_{\text {Best } n-v_{1} \text { alternatives for } 1}}^{\text {Veto }} \cap \underbrace{\left\{x \in A \mid \# L\left(x, \succ_{2}\right) \geq v_{2}\right\}}_{\text {Best } n-v_{2} \text { alternatives for } 2} .
$$

The Pareto-and-veto rule $\mathrm{PV}_{v}$ picks all Pareto efficient alternatives with a lower-contour set at least as large as $v_{i}$ for every player $i$. As an illustration, Table 1 fully describes the outcome of $\mathrm{PV}_{(1,1)}$ in the case of three alternatives. In the table, lines represent the preferences of player 1 and the columns the preferences of player 2, where for short $a b c$ stands for $a \succ_{i} b \succ_{i} c$ and so on. 
Our first observation is that $\mathrm{PV}_{v}$ is non empty when $v_{1}+v_{2} \leq n$. To see this, just observe that eliminating $n$ alternatives at most, out of $n+1$, leaves at least one, say $a$. If $a$ is Pareto efficient, we are done. If not, $a$ is Pareto-dominated by some $a^{\prime} \in \mathrm{PO}_{v}$, but since $a^{\prime}$ is at least as good as $a$ for player $i, a$ is still among her $n-v_{i}$ best alternatives. As soon as $v_{1}+v_{2}$ is at least $n+1$, the example of completely opposed preferences shows that $\mathrm{PV}_{v}$ can be empty.

\subsection{Implementation of $\mathrm{PV}_{v}$}

We now turn to the implementation of $\mathrm{PV}_{v}$ : the strike mechanism with veto vector $v$ Nashimplements the Pareto-and-veto rule with the same veto vector.

Theorem 1. Let the domain $\kappa$ satisfy $\boldsymbol{B E B}$ in $\Delta^{\mathrm{uni}}$, the range of the strike mechanisms. For any $v \in\{0, \ldots, n\}^{2}$ with $v_{1}+v_{2}=n$, the $S C R \mathrm{PV}_{v}$ is Nash-implementable by a DE-mechanism. In particular $\mathrm{PV}_{v}$ is Nash-implementable by the strike mechanism $\mu_{v}$.

Proof. (i) In order to check the inclusion $\operatorname{PV}_{v}(\succ) \supseteq \bigcup_{m \in \mathcal{N}^{\mu_{v}}\left(\succeq^{*}\right)} \operatorname{SUPP}\left(\mu_{v}(m)\right)$, consider any equilibrium $m$. By Proposition 2 the support of $\mu_{v}(m)$ is a singleton $\{x\}$. Because player $i$ can always veto her worst $v_{i}$ alternatives in the mechanism $\mu_{v}$ any best response outcome, and thus any equilibrium outcome $x$ is such that $\# L\left(x, \succ_{i}\right) \geq v_{i} \forall i \in N$. So $x$ satisfies the veto conditions in the definition of PV. It remains to show that $x \in \mathrm{PO}(\succ)$. Suppose not, i.e., there exists $y \in A$ with $y \succ_{i} x$ for all $i \in N$. Since $\mu_{v}(m)=\{x\}$, we have $m_{1} \cap m_{2}=\emptyset$. Thus, $y \in m_{i}$ for some $i \in N$, say $i=1$, without loss of generality. It follows that $y \in A \backslash m_{2}$ and thus $\{y\} \in g_{v}\left(\mathcal{M}_{1}, m_{2}\right)$. Therefore, $\mu_{v}\left(m_{1}^{\prime}, m_{2}\right)=\{y\}$ for some $m_{1}^{\prime}$ and as $\{y\} \succ_{1}^{*} \mu_{v}(m)=\{x\}$, we contradict $m \in \mathcal{N}^{\mu_{v}}\left(\succeq^{*}\right)$.

(ii) For the reverse inclusion, take $x \in \mathrm{PV}_{v}(\succ)$. Because $x$ is Pareto-optimal, any of the $n$ other alternatives is either strictly better than $x$ for one and only one player or strictly worse for both. So counting these $n=v_{1}+v_{2}$ alternatives we obtain:

$$
v_{1}+v_{2}=\# U\left(x, \succ_{1}\right)+\# U\left(x, \succ_{2}\right)+\#\left(L\left(x, \succ_{1}\right) \cap L\left(x, \succ_{2}\right)\right) .
$$

By definition of $\mathrm{PV}_{v}, v_{1} \leq \# L\left(x, \succ_{1}\right)=n-\# U\left(x, \succ_{1}\right)$. Therefore $v_{2} \geq \# U\left(x, \succ_{1}\right)$, which means that player 2 has enough vetoes to block all the alternatives that player 1 strictly prefers to $x$. The same holds for player 1 with respect to player 2. Writing Equation (1) as:

$$
\left[v_{1}-\# U\left(x, \succ_{2}\right)\right]+\left[v_{2}-\# U\left(x, \succ_{1}\right)\right]=\#\left(L\left(x, \succ_{1}\right) \cap L\left(x, \succ_{2}\right)\right),
$$

one can see that it is possible to have players 1 and 2 respectively veto $v_{1}-\# U\left(x, \succ_{2}\right)$ and $v_{2}-\# U\left(x, \succ_{1}\right)$ different alternatives in $L\left(x, \succ_{1}\right) \cap L\left(x, \succ_{2}\right)$, so that all $n$ alternatives are vetoed by one player or the other.

Let $m_{1}$ and $m_{2}$ be such strategies. We now prove that, under $\mathbf{B E B}, m_{1}$ is a strict best response to $m_{2}$. To this end, recall that $U\left(x, \succ_{1}\right) \subseteq m_{2}$ : any alternative strictly preferred by player 1 to $x$ is vetoed by player 2 . So when player 1 deviates to $m_{1}^{\prime} \in \mathcal{M}_{1}$, the support $A \backslash\left(m_{1}^{\prime} \cup m_{2}\right)$ of the outcome lottery excludes $U\left(x, \succ_{1}\right)$. Because of the constraints on the number of vetoes, $\mu\left(m_{1}^{\prime}, m_{2}\right)=\{x\}$ is impossible for $m_{1}^{\prime} \neq m_{1}$. Therefore, for player 1 , the support of $\mu\left(m_{1}^{\prime}, m_{2}\right)$ either contains only alternatives that are strictly worse than $x$, or contains $x$ and some other alternatives that all are worse than $x$. By BEB, player 1 strictly prefers $\{x\}$ to such outcomes, so $m_{1}$ is the unique best response to $m_{2}$. The same holds for the other player, so that we proved that $x$ is an equilibrium outcome. 
Remark that in Theorem 1 the domain is $\Delta^{u n i}$ since the strike mechanisms break ties through uniform lotteries. However, as discussed right after Definition 1, the uniformity can be replaced by any probability distribution.

\subsection{On the necessity of vetoes}

This section presents a counterpart to Theorem 1 by showing that, under a mild richness condition of the domain of preference extensions over lotteries, any Pareto efficient SCR that is Nash-implementable through a DE mechanism is a Pareto-and-veto rule.

Theorem 2. Let $f$ be a Pareto efficient SCR that is Nash-implementable by a DE mechanism $\mu$ on a domain $\kappa$. Let the domain $\kappa$ satisfy $\boldsymbol{B E B}$ and $\boldsymbol{P R E X}$ in the range of $\mu$. Then $f=\mathrm{PV}_{v}$ for some $v \in\{0, \ldots, n\}^{2}$ with $v_{1}+v_{2}=n$.

To prepare for the proof we let, for each player $i$,

$$
\operatorname{veto}(\mu, i)=\left\{X \in \mathcal{A} \mid \exists m_{i} \in \mathcal{M}_{i} \text { s.t. } \operatorname{SUPP}\left(\mu\left(m_{i}, m_{j}\right)\right) \cap X=\emptyset \text { for all } m_{j} \in \mathcal{M}_{j}\right\},
$$

denote the veto set for player $i$. When $X \in \operatorname{veto}(\mu, i)$, we say that player $i$ has veto power over the set $X$ of alternatives, i.e., she has a strategy that ensures that no alternative in this set belongs to the support of the outcome independently of the strategy of her opponent.

The next lemma, whose proof is included in Appendix A, shows that we can restrict attention to mechanisms that are "neutral on their vetoes". A mechanism $\mu$ is neutral on its vetoes for player $i$ iff for any $X \in \mathcal{A}$ and any permutation $\rho: A \rightarrow A, X \in \operatorname{veto}(\mu, i) \Longleftrightarrow \rho(X) \in$ $\operatorname{veto}(\mu, i)$. This does not mean that any player has any veto power (veto $(\mu, i)$ can be empty) nor does it mean that the mechanism $\mu$ is neutral ( $\mu$ does not have to treat alternatives in a symmetric way), it just means that if a set with a given cardinality belongs to veto $(\mu, i)$ then any other set with the same cardinality belongs to veto $(\mu, i)$ as well. Note that a player with veto power over $X$ has also veto power over any $X^{\prime} \subset X$. Hence, the veto set for player $i$ can be written as:

$$
\operatorname{veto}(\mu, i)=\left\{X \in \mathcal{A} \mid \# X \leq v_{i}\right\}
$$

where the integer $v_{i}$ stands for the cardinality of the highest cardinality set over which $i$ has veto power.

Lemma 2. Under the hypothesis of Theorem 2, $\mu$ is neutral on its vetoes for both players.

We can now proceed to a complete proof of the Theorem.

Proof of Theorem 2. (1) We first establish the existence of $v$ such that $f \subseteq \mathrm{PV}_{v}$. Let $\mathcal{M}$ be the joint message space of $\mu$ that DE-implements $f$. By assumption, the domain satisfies PREX and BEB. Take any preference profile $\succ=\left(\succ_{1}, \succ_{2}\right)$ and any $x \in f(\succ)$. Let $\succeq^{*}=\left(\succeq_{1}^{*}, \succeq_{2}^{*}\right)$ be a priority extension of $\succ$ for $x$ that exists due to PREX. Thus, for all $p, q \in \mu(\mathcal{M})$ and for $i=1,2$, if $p\left[U\left(x, \succ_{i}\right)\right]>0$ and $q\left[U\left(x, \succ_{i}\right)\right]=0$ then $p \succ_{i}^{*} q$. By assumption, $\mu$ admits a Nash equilibrium $\left(m_{1}, m_{2}\right)$ at $\succeq^{*}$ with $\mu\left(m_{1}, m_{2}\right)=\{x\}$. By the definition of an equilibrium, player 2 has no better response to $m_{1}$ than $m_{2}$. However, under $\succeq_{2}^{*}$, a deviation $m_{2}^{\prime}$ is profitable for player 2 iff $\operatorname{SUPP}\left(\mu\left(m_{1}, m_{2}^{\prime}\right)\right) \cap U\left(\succ_{2}, x\right) \neq \emptyset$. Therefore:

$$
\forall m_{2} \in \mathcal{M}_{2}, \operatorname{SUPP}\left(\mu\left(m_{1}, m_{2}\right)\right) \cap U\left(\succ_{2}, x\right)=\varnothing
$$


and likewise for player 1 . In other words, $m_{1}$ makes the set $U\left(\succ_{2}, x\right)$ unattainable for player 2 under $\mu$. Thus $m_{1}$ gives player 1 veto power on the set $U\left(\succ_{2}, x\right)$, and likewise for player 2 . Hence,

$$
U\left(\succ_{2}, x\right) \in \operatorname{veto}(\mu, 1), U\left(\succ_{1}, x\right) \in \operatorname{veto}(\mu, 2) .
$$

From Lemma 2, if a player has veto power on some set, she has also veto power on any set of the same cardinality. Let $v_{i}$ be the largest number of outcomes that $i$ can veto. For the mechanism to be well-defined, one needs $v_{1}+v_{2} \leq n$, so that not all the $n+1$ alternatives can be vetoed simultaneously. Now consider the case when the preference profile consists of opposed preferences: we have that $U\left(\succ_{1}, x\right) \cap U\left(\succ_{2}, x\right)=\emptyset$. The existence of a deterministic equilibrium (an equilibrium with a singleton outcome) in that case shows that $v_{1}+v_{2} \geq n$. Hence $v_{1}+v_{2}=n$.

Now, we claim that at $\succeq^{*}$, an equilibrium outcome is not among the $v_{i}$ worse alternatives for any player $i$. The claim implies that $f \subseteq \mathrm{PV}_{v}$, since equilibrium outcomes depend only on $\succ$.

To prove the claim, let $m \in \mathcal{N}^{\mu}\left(\succeq^{*}\right)$ be an equilibrium and $x$ its outcome. Assume that $x$ is among the $v_{1}$ worst alternatives of player 1 . As previously argued, player 1 has a veto power of $v_{1}$. Let $m_{1}^{\prime}$ denote the strategy of player 1 that vetoes his worst $v_{1}$ alternatives. For any $m_{2} \in \mathcal{M}_{2}$, the support of $\mu\left(m_{1}^{\prime}, m_{2}\right)$ is included in player 1's top $n+1-v_{1}$ alternatives. However, due to PREX, player 1 prefers any such lottery to $x$, which proves that player 1 has a profitable deviation and thus $m$ is not an equilibrium.

(2) For this $v$, we now prove the reverse inclusion. Given $\succ=\left(\succ_{1}, \succ_{2}\right)$, let $x \in \mathrm{PV}_{v}(\succ)$. Consider the profile $\succ^{\prime}$ defined as follows.

Label the $n+1$ alternatives in two ways: $a_{n+1} \succ_{1} a_{n} \succ_{1} \ldots \succ_{1} a_{1}$ and $b_{n+1} \succ_{2} b_{n} \succ_{2} \ldots \succ_{2}$ $b_{1}$. Write $a_{w_{1}}=b_{w_{2}}=x$. The veto conditions in the definition of $\mathrm{PV}_{v}$ are that $w_{1}>v_{1}$ and $w_{2}>v_{2}$, which implies that:

$$
\begin{aligned}
& a_{n+1} \succ_{1} \ldots \succ_{1} a_{w_{1}}=x \succ_{1} \ldots \succ_{1} a_{v_{1}} \succ_{1} \ldots \succ_{1} a_{1}, \\
& b_{n+1} \succ_{2} \ldots \succ_{2} b_{w_{2}}=x \succ_{2} \ldots \succ_{2} b_{v_{2}} \succ_{2} \ldots \succ_{2} b_{1} .
\end{aligned}
$$

The preference $\succ_{1}^{\prime}$ is obtained by lowering the ranks of all those, among the alternatives $a_{v_{1}+1}, \ldots$, $a_{w_{1}-1}$, which are preferred to $x$ by the other player, player 2 . If $w_{1}=v_{1}+1$ we simply let $\succ_{1}^{\prime}=\succ_{1}$. If $w_{1} \geq v_{1}+2$, consider the set

$$
H_{1}=\left\{a_{v_{1}+1}, \ldots, a_{w_{1}-1}\right\} \cap\left\{b_{w_{2}+1}, \ldots, b_{n+1}\right\}
$$

and observe that

$$
\# H_{1} \leq n-w_{2} \leq n-v_{2}=v_{1} .
$$

Starting from $\succ_{1}$, we define $\succ_{1}^{\prime}$ by switching in the ranking the first elements $a_{1}, \ldots a_{\# H_{1}}$ with the elements of $H_{1}$, where $a_{1}$ is switched with the most preferred element of $H_{1}$ of player $1, a_{2}$ is switched with the second most preferred element of $H_{1}$ of player 1 and so on...

We now claim that if $x \in f\left(\succ_{1}^{\prime}, \succ_{2}\right)$ then $x \in f(\succ)$. Let $\mu$ DE-implement $f$. If $x \in f\left(\succ_{1}^{\prime}\right.$ ,$\left.\succ_{2}\right)$, there exists a pure strategy equilibrium $\left(m_{1}^{\prime}, m_{2}^{\prime}\right)$ for the game with preferences $\left(\succ_{1}^{\prime}, \succ_{2}\right)$ with $\{x\}=\mu\left(m_{1}^{\prime}, m_{2}^{\prime}\right)$. With the initial preferences $\left(\succ_{1}, \succ_{2}\right), m_{2}$ is also a best response since player 2 does not change her preference, and $m_{1}^{\prime}$ is also a best response for player 1 because her preferences differ only below $x$. As previously argued, $m_{2}$ gives player 2 veto power on the set $U\left(\succ_{1}, x\right)$. Since $U\left(\succ_{1}^{\prime}, x\right)=U\left(\succ_{1}, x\right)$ by construction, it follows that the support of any lottery that player 1 can attain given $m_{2}$ is included in $A \backslash U\left(\succ_{1}, x\right)$. Hence, due to BEB, $m_{1}$ is a best 
response for player 1 since $\mu\left(m_{1}, m_{2}\right)=\{x\}$. Therefore this equilibrium for $\left(\succ_{1}^{\prime}, \succ_{2}\right)$ is also an equilibrium for $\left(\succ_{1}, \succ_{2}\right)$, that is: $x \in f\left(\succ^{\prime}\right) \Longrightarrow x \in f(\succ)$.

The same construction for player 2 yields the preference profile $\succ^{\prime \prime}=\left(\succ_{1}^{\prime}, \succ_{2}^{\prime}\right)$ with the property:

$$
x \in f\left(\succ^{\prime \prime}\right) \Longrightarrow x \in f(\succ)
$$

But notice that, by construction of $\succ_{1}^{\prime}$, all the alternatives $y$ such that $y \succ_{2} x$ are now among the $v_{1}$ worse alternatives according to $\succ_{1}^{\prime}$. Therefore $x$ is the preferred alternative, according to $\succ_{2}^{\prime}$, among the alternatives in the intersection of the top $n-v_{1}$ alternatives for player 1 and $n-v_{2}$ alternatives for player 2 in $\succ^{\prime}$. Since the same is true for the other player, we find that $x$ is the unique Pareto optimum in the alternatives among the top $n-v_{1}$ alternatives for player 1 and the top $n-v_{2}$ alternatives for player 2 in $\succ^{\prime \prime}$. Since $f$ itself is assumed to be efficient and is selecting in $\mathrm{PV}_{v}$, we obtain that $f\left(\succ^{\prime \prime}\right)=\{x\}$. From (2) it follows that $x \in f(v)$ as requested.

Theorem 2 shows the existence of a strong link between implementation through DE mechanisms and veto power. Indeed, it shows that under the conditions BEB and PREX, a SCR has to admit some veto structure in order to be both Pareto efficient and Nash-implementable. For the sake of clarity, the following corollary summarizes the main message of Theorems 1 and 2.

Corollary 1. Suppose that a domain $\kappa$ satisfies $\boldsymbol{B E B}$ and PREX in $\Delta^{\text {uni }}$. The following are equivalent:

1. $f$ is a Pareto efficient SCR that is Nash-implementable by a $\mathbf{D E}$ mechanism on $\kappa$.

2. $f=P V_{v}$ for some $v \in\{0, \ldots, n\}^{2}$ with $v_{1}+v_{2}=n$.

Theorem 2 is related to the impossibility result by Hurwicz and Schmeidler (1978) in the following sense. Hurwicz and Schmeidler (1978) show that the only SCRs which are both Pareto efficient and Nash-implementable (through a deterministic mechanism) are the dictatorial ones. Note that a dictatorial SCR corresponds to $\mathrm{PV}_{v}$ with $v=(n, 0)$ (if player 1 is the dictator) or $v=$ $(0, n)$ (if player 2 is the dictator). Our theorem shows that by allowing lotteries as off-equilibrium punishments, the Pareto-and-veto rules appear as a class of intermediate and, interestingly, non dictatorial SCRs.

Note that $\mathrm{PV}_{v}$ is neutral for any $v \in\{0 \ldots, n\}^{2}$ and that it is anonymous if and only if $v_{1}=v_{2}$. Thus, under the assumptions of Theorem 2, the following observations trivially follow. With an odd number of alternatives, an anonymous, neutral and Pareto efficient SCR $f$ is Nashimplementable by a DE mechanism iff $f$ is a Pareto-and-veto rule with $v_{1}=v_{2}$. On the contrary, with an even number of alternatives, there exist no anonymous, neutral and Pareto efficient SCR that is Nash-implementable by a DE mechanism.

We complete this section by providing three examples in Appendix B. The first one illustrates the necessity of PREX in Theorem 2: On a domain that satisfies all our assumptions except PREX, we build a Pareto efficient SCR that is implemented by a DE mechanism but is not a $P V_{v}$ with $v_{1}+v_{2}=n$. The second and third examples clarify the role of Pareto efficiency: the domain satisfies BEB and PREX; the mechanism is DE but the implemented SCR is not Pareto efficient. 


\section{Connections with two-player Nash implementation theory}

A complete characterization of Nash-implementable SCRs with two players was independently achieved by both Moore and Repullo (1990) and Dutta and Sen (1991). In order to clarify the connection between our results and these characterizations, we quote condition $\beta$ of Dutta and Sen (1991) (whose equivalent version is called condition $\mu_{2}$ in Moore and Repullo (1990)) which is necessary and sufficient for a SCR to be Nash-implementable with two players.

For any $i \in N$, let $\widetilde{L}\left(x, \succ_{i}\right)=L\left(x, \succ_{i}\right) \cup\{x\}$ be the weak lower contour set of $x \in A$ at $\succ_{i} \in \mathcal{L}_{A}$ and $M\left(C, \succ_{i}\right)=\left\{a \in C \mid a \succ_{i} c \forall c \in C \backslash\{a\}\right\}$ be the singleton set containing the maximal elements of $C \subseteq A$ with respect to $\succ_{i} \in \mathcal{L}_{A}$.

Definition 3. A SCR $f$ satisfies condition $\beta$ iff there exists a set $A^{*}$ which contains the range of $f$, and for each $i \in N, \succ \in \mathcal{L}_{A}^{2}$ and $a \in f(\succ)$, there exists a set $C_{i}(a, \succ) \subseteq A^{*}$, with $x \in C_{i}(a, \succ$ )$\subseteq \widetilde{L}\left(a, \succ_{i}\right)$ such that for all $\succ^{\prime} \in \mathcal{L}_{A}^{2}$, we have:

(i) (a) for all $b \in f\left(\succ^{\prime}\right), C_{1}(a, \succ) \cap C_{2}\left(b, \succ^{\prime}\right) \neq \emptyset$.

(b) Moreover, there exists $x \in C_{1}(a, \succ) \cap C_{2}\left(b, \succ^{\prime}\right)$ such that if for some $\succ^{\prime \prime} \in \mathcal{L}_{A}^{2}, x \in$ $M\left(C_{1}(a, \succ), \succ_{1}^{\prime \prime}\right) \cap M\left(C_{2}\left(b, \succ^{\prime}\right), \succ_{2}^{\prime \prime}\right)$, then $x \in f\left(\succ^{\prime \prime}\right)$.

(ii) if $a \notin f\left(\succ^{\prime}\right)$, then there exist $j \in N$ and $b \in C_{j}(a, \succ)$ such that $b \notin \widetilde{L}\left(a, \succ^{\prime}\right)$.

(iii) $M_{i}\left(C_{i}(a, \succ) \backslash\{a\}, \succ^{\prime}\right) \cap M_{j}\left(A^{*}, \succ^{\prime}\right) \subseteq f\left(\succ^{\prime}\right) \forall i \in N$ and $j \neq i$.

(iv) $M\left(A^{*}, \succ_{1}^{\prime}\right) \cap M\left(A^{*}, \succ_{2}^{\prime}\right) \subseteq f\left(\succ^{\prime}\right)$.

Without restrictions on the domain of preferences, only dictatorial SCRs satisfy condition $\beta$ (in line with the impossibility results of Hurwicz and Schmeidler (1978) and Maskin (1999)). As Moore and Repullo (1990) notes, parts (ii), (iii) and (iv) of condition $\beta$ are necessary and sufficient for Nash implementation with three or more players. Among these, part (ii) corresponds to Maskin monotonicity; part (iv) is a unanimity condition while part (iii) is a relaxation of the no-veto power condition. On the other hand, condition $(i)$ is central for the situation with two players. However, $(i)(a)$, which has been referred to as a self-selection constraint or simply intersection property (see Abreu and Sen (1991) for a discussion) turns out to be a critical condition for different implementation concepts such as virtual implementation (Abreu and Sen (1991)) or implementation with partially honest players (Dutta and Sen (2012)). Busetto and Codognato (2009) has shown that the different parts of condition $\beta$ exhibit problems of logical dependence.

For the sake of precision, we introduce the definitions of the intersection property and Maskin monotonicity formally, respectively implied by conditions $\beta(i)(a)$ and $\beta(i i)$.

Definition 4. A SCR $f$ satisfies the intersection property (IP) iff for all $\succ, \succ^{\prime} \in \mathcal{L}_{A}^{2}$ and $x, y \in A$ with $x \in f(\succ)$ and $y \in f\left(\succ^{\prime}\right)$, we have $\widetilde{L}\left(x, \succ_{i}\right) \cap \widetilde{L}\left(y, \succ_{j}^{\prime}\right) \neq \emptyset$ for any $i \in N \backslash\{j\}$.

Definition 5. A $\underset{\sim}{\operatorname{SCR} f}$ satisfies Maskin monotonicity (MM) iff for all $\succ, \succ^{\prime} \in \mathcal{L}_{A}^{2}$ and $x \in A$ with $\widetilde{L}\left(x, \succ_{i}\right) \subseteq \widetilde{L}\left(x, \succ_{i}^{\prime}\right) \forall i \in N$, we have $x \in f(\succ) \Longrightarrow f\left(\succ^{\prime}\right)$.

We first observe that the necessity of $M M$ and $I P$ prevails when DE mechanisms are used. Theorem 2 has shown that when the domain satisfies BEB and PREX, a Pareto efficient SCR that is Nash-implementable by a DE mechanism is a Pareto-and-veto rule. Thus, the necessity of $M M$ and $I P$ for DE mechanisms can be seen by establishing that Pareto-and-veto rules satisfy both conditions: 
Proposition 3. For any veto vector $v$, the Pareto-and-veto rule $\mathrm{PV}_{v}$ satisfies IP and MM.

Proof. In order to check $I P$, for any veto vector $v$, take any pair $\succ, \succ^{\prime} \in \mathcal{L}_{A}^{2}$ and any $x \in \mathrm{PV}_{v}(\succ)$ and $y \in \operatorname{PV}_{v}\left(\succ^{\prime}\right)$. By definition of $\mathrm{PV}_{v}, \# \widetilde{L}\left(x, \succ_{i}\right) \geq v_{i}+1$ and $\# \widetilde{L}\left(y, \succ_{j}\right) \geq v_{j}+1$ for $j \neq i$. Yet, since $v_{1}+v_{2}=n$, it follows that $\# \widetilde{L}\left(x, \succ_{i}\right)+\# \widetilde{L}\left(y, \succ_{j}\right) \geq n+2$ and hence $\widetilde{L}\left(x, \succ_{i}\right) \cap \widetilde{L}\left(y, \succ_{j}^{\prime}\right.$ )$\emptyset$, which shows that $I P$ holds.

In order to check $M M$, for any veto vector $v$, take any $\succ \in \mathcal{L}_{A}^{2}$ and any $x \in \operatorname{PV}_{v}(\succ)$. Let $\succ^{\prime} \in \mathcal{L}_{A}^{2}$ be some profile with $L\left(x, \succ_{i}\right) \subseteq L\left(x, \succ_{i}^{\prime}\right) \forall i \in N$. Note that $x \in \mathrm{PO}(\succ)$ implies that $x \in$ $\mathrm{PO}\left(\succ^{\prime}\right)$. Moreover, \#L $\left(x, \succ_{i}^{\prime}\right) \geq \# L\left(x, \succ_{i}\right)$ for each $i \in N$ (by construction of $\left.\succ^{\prime}\right)$ and \#L $\left(x, \succ_{i}\right.$ ) $\geq v_{i} \forall i \in N$ (by the definition of $\mathrm{PV}_{v}$ ). Thus $x \in \mathrm{PV}_{v}\left(\succ^{\prime}\right)$, as desired.

Interestingly, $M M$ and $I P$ pave the way towards a full characterization of the class of Paretoand-veto rules.

Definition 6. Under a SCR $f$, player $i$ has veto power over the set $X \subset A$ at the profile $\succ \in \mathcal{L}_{A}^{2}$ iff for any $Y \subseteq X$, if $z \succ_{i} y \forall z \in A \backslash Y, \forall y \in Y$ then $f(\succ) \cap Y=\emptyset$. When $i$ has veto power over $X$ for any $\succ \in \mathcal{L}_{A}^{2}$, we say that $i$ has veto power over $X$ under $f$.

Remark that if player $i$ can veto $X$ under $f$, she can also veto any subset $Y$ of $X$ whenever $Y$ consists of her least preferred alternatives. When player $i$ does not have veto power over $X$ under $f$, this means that there exists a profile $\succ$ in which $X$ are the least preferred alternatives of player $i$ in $\succ_{i}$ and such that $f(\succ) \cap X \neq \emptyset$.

Definition 7. We say that a SCR $f$ is neutral-on-its-vetoes iff whenever $f$ gives veto power to player $i$ over a set $X, f$ gives veto power to $i$ over every set $Y$ with $\# Y=\# X$.

Note that when $f$ is neutral-on-its-vetoes, the veto power of player $i$ can be expressed by an integer $v_{i} \in\{0, \ldots, n\}$ which is the cardinality of the largest set that $i$ can veto.

Proposition 4. If $f$ satisfies IP and is Pareto efficient and neutral-on-its-vetoes, then $f \subseteq \mathrm{PV}_{v}$.

Proof. Take some $f$ which is neutral-on-its-vetoes, satisfies $I P$ and is Pareto efficient. Assume that at some profile $\succ, x \in f(\succ)$ with $\# L\left(x, \succ_{1}\right)=k$ for $k=0, \ldots, n$. IP implies that any profile $\succ^{\prime}, f\left(\succ^{\prime}\right) \subseteq A \backslash L\left(x, \succ_{2}^{\prime}\right)$. Therefore, if player 1 cannot prevent $x$ at $f(\succ)$, then player 2 can ensure at any profile that $L\left(x, \succ_{2}^{\prime}\right) \geq n-k$. Again, this observation can be generalized since $f$ neutral-on-its-vetoes: $v_{1}=k$ implies that $v_{2}=n-k$.

Since $f$ is Pareto-efficient and only selects alternatives such that $v_{1}+v_{2}=n$, it follows that $f \subseteq \mathrm{PV}_{v}$ as required.

Proposition 5. For any veto vector $v, f \subseteq \mathrm{PV}_{v}$ satisfies $M M$ if and only if $f=\mathrm{PV}_{v}$

Proof. Note that the proof is immediate if either $v_{1}=n$ or $v_{2}=n$ since, in both cases, $\mathrm{PV}_{v}$ is singleton valued for each preference profile $\succ$. Thus, $f \subseteq \mathrm{PV}_{v}$ directly implies that $f=\mathrm{PV}_{v}$ and hence is $M M$. We assume that $0<v_{1}, v_{2}<n$ in the sequel of the proof.

We show first that for any $f \subseteq \mathrm{PV}_{v}$, any $\succ \in \mathcal{L}_{A}^{2}$ and any $x \in \mathrm{PO}(\succ)$, if \# $L\left(x, \succ_{i}\right)=v_{i} \forall i \in N$, then $f(\succ)=\{x\}$. Take any $f \subseteq \mathrm{PV}_{v}$, any $\succ \in \mathcal{L}_{A}^{2}$ and any $x \in \mathrm{PO}(\succ)$ with $\# L\left(x, \succ_{i}\right)=v_{i}$ $\forall i \in N$. Assume by contradiction that there is some $y \in f(\succ)$ with $y \in A \backslash\{x\}$. Since $f \subseteq \operatorname{PV}_{v}$, 
$\# L\left(y, \succ_{i}\right) \geq v_{i} \forall i \in N$, and as $\# L\left(x, \succ_{i}\right)=v_{i} \forall i \in N$, it follows that $\# L\left(y, \succ_{i}\right)>v_{i} \forall i \in N$. But this implies that $y \succ_{i} x \forall i \in N$, contradicting $x \in \mathrm{PO}(\succ)$. Hence, since $f(\succ)$ is non-empty, $f(\succ)=\{x\}$ as wanted.

We now show that for any $\succ \in \mathcal{L}_{A}^{2}$ and any $x \in \mathrm{PV}_{v}(\succ)$, if $f$ is $\mathrm{MM}$ and $f \subseteq \mathrm{PV}_{v}$, then $x \in$ $f(\succ)$. For each $x \in A$ and each veto vector $v$, let $B_{v}^{x}=\left\{\succ \in \mathcal{L}_{A}^{2} \mid x \in \operatorname{PO}(\succ)\right.$ with $\# L\left(x, \succ_{i}\right)=$ $\left.v_{i} \forall i \in N\right\}$. Since the preferences are unrestricted, for any partition $(X, Y)$ of $A \backslash\{x\}$ with \#X=vi and $\# Y=v_{j}$, there is some $\succ \in B_{v}^{x}$ such that $L\left(x, \succ_{i}\right)=X$ and $L\left(x, \succ_{j}\right)=Y$.

As shown before, we know that for any $f \subseteq \mathrm{PV}_{v}$ and any $\succ \in B_{v}^{x}, f(\succ)=\{x\}$ with $\widetilde{L}\left(x, \succ_{1}\right.$ )$\cup \widetilde{L}\left(x, \succ_{2}\right)=A$ since $x \in \mathrm{PO}(\succ)$ with $\# L\left(x, \succ_{1}\right)+\# L\left(x, \succ_{2}\right)=n\left(\right.$ since $\left.v_{1}+v_{2}=n\right)$.

Consider now any profile $\succ^{\prime}$ with $x \in \mathrm{PV}_{v}\left(\succ^{\prime}\right)$. Assume by contradiction that $x \notin f\left(\succ^{\prime}\right)$. Since $x \in \mathrm{PV}_{v}\left(\succ^{\prime}\right)$, it follows that $x \in \mathrm{PO}\left(\succ^{\prime}\right)$ and $\# L\left(x, \succ_{i}^{\prime}\right) \geq v_{i}$ for all $i \in N$. Note that there is at least some strict inequality since otherwise $\succ^{\prime} \in B_{v}^{x}$ and hence $x \in f\left(\succ^{\prime}\right)$, a contradiction. Since $x \in \operatorname{PO}\left(\succ^{\prime}\right), L\left(x, \succ_{1}^{\prime}\right) \cup L\left(x, \succ_{2}^{\prime}\right) \cup\{x\}=A$ whereas $\# L\left(x, \succ_{1}^{\prime}\right)+\# L\left(x, \succ_{2}^{\prime}\right)>n$. Since $L\left(x, \succ_{1}^{\prime}\right) \cup L\left(x, \succ_{2}^{\prime}\right) \cup\{x\}=A$, it follows that:

$$
\#\left(L\left(x, \succ_{1}^{\prime}\right) \backslash L\left(x, \succ_{2}^{\prime}\right)\right)+\#\left(L\left(x, \succ_{2}^{\prime}\right) \backslash L\left(x, \succ_{1}^{\prime}\right)\right)+\#\left(L\left(x, \succ_{1}^{\prime}\right) \cap L\left(x, \succ_{2}^{\prime}\right)\right)=n=v_{1}+v_{2},
$$

where $L\left(x, \succ_{1}^{\prime}\right) \cap L\left(x, \succ_{2}^{\prime}\right)$ denote the set of alternatives that $x$ Pareto dominates. Since \#L $\left(x, \succ_{1}^{\prime}\right.$ )$+\# L\left(x, \succ_{2}^{\prime}\right)>n$, note that $L\left(x, \succ_{1}^{\prime}\right) \cap L\left(x, \succ_{2}^{\prime}\right) \neq \emptyset$.

Since $\#\left(L\left(x, \succ_{1}^{\prime}\right) \backslash L\left(x, \succ_{2}^{\prime}\right)\right) \leq v_{1}{ }^{10}$ and $\#\left(L\left(x, \succ_{2}^{\prime}\right) \backslash L\left(x, \succ_{1}^{\prime}\right)\right) \leq v_{2}$, we can find a partition $(X, Y)$ of $A \backslash\{x\}$ with

$X \subseteq L\left(x, \succ_{1}^{\prime}\right)$ and $Y \subseteq L\left(x, \succ_{2}^{\prime}\right)$ with $X \cap Y=\emptyset, \# X=v_{1}$ and $\# Y=v_{2}$.

It follows that there is some $\succ^{*} \in B_{v}^{x}$ with $\widetilde{L}\left(x, \succ_{i}^{*}\right) \subseteq \widetilde{L}\left(x, \succ_{i}^{\prime}\right)$ since $\widetilde{L}\left(x, \succ_{i}^{*}\right)=v_{i}+1$ and $\widetilde{L}\left(x, \succ_{i}^{\prime}\right) \geq v_{i}+1 \forall i \in N$. Moreover, $x \in f\left(\succ^{*}\right)$ since $\succ^{*} \in B_{v}^{x}$. Hence $M M$ implies that $x \in$ $f\left(\succ^{\prime}\right)$, as desired.

We have shown that any alternative that could be selected by a Pareto-and-veto rule is selected by any $M M$ sub-correspondence which shows the desired result.

We are now in the position to characterize the class of Pareto-and-veto rules by the conditions of $I P, M M$, Pareto efficiency and neutrality-on-its vetoes. These conditions are independent as shown in the appendix.

Theorem 3. A SCR $f$ satisfies IP and is neutral-on-its vetoes, MM and Pareto efficient if and only if $f$ is a Pareto-and-veto rule.

Proof. Take some $f$ that satisfies $I P$ and is neutral-on-its vetoes, $M M$ and Pareto efficient. Proposition 4 implies that $f$ is a sub-correspondence of a Pareto-a-veto rule. Moreover, Proposition 5 shows that the only $M M$ subcorrespondence of a Pareto-a-veto rule is the Pareto-and-veto rule itself, proving the if claim. The converse implication follows directly from Proposition 3.

To conclude our comments on the classical Moore-Repullo-Dutta-Sen characterization we point precisely which condition, in this result, is not satisfied by the Pareto-and-veto rules.

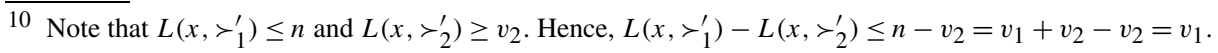


Proposition 6. For any veto vector $v$, the Pareto-and-veto rule $\mathrm{PV}_{v}$ fails condition $\beta(i)(b)$

Proof. We provide a proof for three alternatives and for the Pareto-and-veto rule with veto vector $v=(1,1)$. It can be easily generalized to any Pareto-and-veto rule and any number of alternatives. Let $\succ=\left(\succ_{1}, \succ_{2}\right)$ and $\succ^{\prime}=\left(\succ_{1}^{\prime}, \succ_{2}^{\prime}\right)$ be two preference profiles such that: (1) $c \succ_{1} a \succ_{1} b$ and $a \succ_{1} b \succ_{1} c$ and (2) $b \succ_{1}^{\prime} a \succ_{1}^{\prime} c$ and $c \succ_{1}^{\prime} b \succ_{1}^{\prime} a$. For these profiles, $\operatorname{PV}_{v}(\succ)=\{a\}$ and $\mathrm{PV}_{v}\left(\succ^{\prime}\right)=\{b\}$. Since $\widetilde{L}\left(a, \succ_{1}\right)=\{a, b\}$ and $\widetilde{L}\left(b, \succ_{2}^{\prime}\right)=\{a, b\}$ as well, we are going to find a violation of condition $\beta(i)(b)$ for profiles $\succ^{\prime \prime}$ that are unanimous $\left(\succ_{1}^{\prime \prime}=\succ_{2}^{\prime \prime}\right)$ and in favor of $c$ $\left(c \succ_{i}^{\prime \prime} a\right.$ and $c \succ_{i}^{\prime \prime} b$ for $\left.i=1,2\right)$, so that $\mathrm{PV}_{v}\left(\succ^{\prime \prime}\right)=\{c\}$

Since $C_{1}(a, \succ)$ and $C_{2}\left(b, \succ^{\prime}\right)$ are subsets of $\{a, b\}$ with a non-empty intersection, as stated by condition $\beta(i)(a)$, the following cases have to be considered:

Case 1: $C_{1}(a, \succ)=C_{2}(b, \succ)$.

In this case, since $\succ^{\prime \prime}$ is unanimous, $M\left(C_{1}(a, \succ), \succ_{1}^{\prime \prime}\right)=M\left(C_{2}\left(b, \succ^{\prime}\right), \succ_{2}^{\prime \prime}\right) \subseteq\{a, b\}$. Therefore $c$ does not belong to the intersection $M\left(C_{1}(a, \succ), \succ_{1}^{\prime \prime}\right) \cap M\left(C_{2}\left(b, \succ^{\prime}\right), \succ_{2}^{\prime \prime}\right)$, in contradiction with $\beta(i)(b)$.

Case 2: $C_{1}(a, \succ)=\{a\}$ and $C_{2}\left(b, \succ^{\prime}\right)=\{a, b\}$, or $C_{1}(a, \succ)=\{a, b\}$ and $C_{2}\left(b, \succ^{\prime}\right)=\{a\}$. Take then $c \succ_{i}^{\prime \prime} a \succ_{i}^{\prime \prime} b$ for $i=1,2$; for this $\succ^{\prime \prime}: M\left(C_{1}(a, \succ), \succ_{1}^{\prime \prime}\right) \cap M\left(C_{2}\left(b, \succ^{\prime}\right), \succ_{2}^{\prime \prime}\right)=\{a\}$, again a contradiction.

Case 3: $C_{1}(a, \succ)=\{b\}$ and $C_{2}\left(b, \succ^{\prime}\right)=\{a, b\}$, or $C_{1}(a, \succ)=\{a, b\}$ and $C_{2}\left(b, \succ^{\prime}\right)=\{b\}$. The same contradiction appears for the unanimous profile such that $c \succ_{i}^{\prime \prime} b \succ_{i}^{\prime \prime} a$.

As a final remark regarding the connections with the literature, we would like to note that Dutta and Sen (1991) show the possibility of two-person implementation through an example (Example 1) with (i) a finite set of alternatives, (ii) players having vNM utilities, (iii) lotteries being allowed. Their example is related to our setting but their possibility for implementation builds on a condition, called Assumption 5.5, which rules out that "individual 2's utility function is the inverse (up to an affine transformation) of 1's utility function". In our framework, the set of preference profiles is the product space $\mathcal{L}_{A}^{2}$ and we impose only restrictions on the preference extensions over lotteries for each player, meaning that individual preferences are independent from each other.

\section{Concluding comments}

This section provides a short review of the two-player implementation problem (see Dutta (2019) for a recent and complete survey) and some concluding comments on the strike mechanisms.

As argued in the introduction, the pioneering works (Hurwicz and Schmeidler (1978) and Maskin (1999)) provide a provocative result: dictatorships are the only Pareto efficient rules that can be Nash implemented. Their proof builds on three key assumptions: $(i)$ the preference domain is universal (any preference profile is allowed) while implementing mechanisms are ( $i$ i) simultaneous and (iii) deterministic. 
The literature has explored the consequences of weakening each of these assumptions. ${ }^{11}$ The first strand relaxes condition (i), Dutta and Sen (1991) and Moore and Repullo (1990) are the key papers in this direction. They identify the domain restrictions under which one can design Pareto efficient and non-dictatorial Nash-implementable rules. While the full characterization is rather complex, the sufficient domain conditions for implementation often rely on the Euclidean space (see Section 5 in Dutta and Sen (1991) for instance); in the current work, we do not impose any structure on the alternatives or the preferences, beyond the assumption that preferences over alternatives are strict.

A second strand is concerned with ( $i i)$, that is, replacing simultaneous with dynamic mechanisms. This literature, in which Moore and Repullo (1988), Abreu and Sen (1991) and Herrero and Srivastava (1992) play a key role, shows that introducing an order of play expands the set of implementable rules with more than two players. No characterization of implementable rules via subgame-perfect or via backward induction is available. By altering the notion of implementation (role-robust implementation), De Clippel et al. (2014) ${ }^{12}$ show that a possibility arises with dynamic vetoes and randomized order of play (see also Barberà and Coelho (2019) who consider the implementation of the fallback-bargaining solution). However, while ex-ante fairness is achieved by randomizing the order of play, ex-post fairness fails. The order of play matters for determining the outcome, creating first, or second, mover advantages. As Moulin (1981) puts it, "voting by veto procedures introduce a strong asymmetry among agents:... the ordering of the agents has a strong influence on the outcome".

The third and final strand of the literature deals with assumption (iii), as does the current work: it explores the consequences of modifying the type of mechanisms. ${ }^{13}$ As mentioned in the introduction, Sanver (2006), Bochet (2007) and Benoît and Ok (2008) exploit the idea of allowing lotteries/awards off-equilibrium. The main idea of these works is that, with at least three players, monotonicity fully characterizes the class of Nash-implementable rules under a domain restriction so that the no-veto power condition is dispensable. This is in line with the results present in this paper in which DE mechanisms expand the set of Nash-implementable rules. Yet, this paper is the first one to consider this idea with two players. This strand of literature is related to the one on virtual implementation, a reformulation of the original implementation problem. A social choice rule is virtually implementable if there exists a game form $G$, such that for all preference profiles $G$ admits a unique equilibrium outcome (a lottery) which is $\varepsilon$-close to the outcome prescribed by the rule at this preference profile and this holds for every $\varepsilon>0$. Following this approach, Matsushima (1988) and Abreu and Sen (1991) provide a strong possibility result: with at least three players, any rule is implementable. With two players, the result is more nuanced but some SCRs are virtually implementable, among which the Pareto-and-veto rule described in the current work. However, under the virtual implementation approach, "any alternative can be the outcome of the game as it receives positive probability in the equilibrium lottery" (Bochet and Maniquet (2010)). In other words, in order to virtually implement a social choice rule, one

\footnotetext{
11 Other approaches have modified the rationality notion, using "partial honesty"; see Dutta and Sen (2012) among others.

12 A classic literature considers sequential voting by veto with many players (see Mueller (1978), Moulin (1981), Bloom and Cavanagh (1986), Felsenthal and Machover (1992) and Anbarci (2006)) where each player is assigned a certain number of vetoes to be distributed freely among the alternatives. See also the rules of $k$-names in Barberà and Coelho (2010).

13 See also the papers on approval voting with two players as Núñez and Laslier (2015) and Laslier et al. (2017). See also Jackson and Sonnenschein (2007) who show that linking decisions (that is, a common decision on several independent problems) can help overcoming incentive constraints in Bayesian collective decision problems.
} 
constructs game forms whose equilibrium outcome at every preference profile is a full-support lottery, arbitrarily close to the outcome prescribed by the social choice rule. This represents a threat to the relevance of these solutions since it involves that socially undesirable alternatives, even with a small probability, can be selected.

Strike mechanisms arise as a solution to the two-person implementation problem. This solution is obtained by altering two key elements of the classic framework: (i) considering mechanisms that allow in equilibrium pure alternatives and off equilibrium lotteries and (ii) restricting efficiency to the ex-post Pareto notion.

Our class of DE mechanisms is a simultaneous version of the dynamic veto mechanisms (see Moulin (1981) and De Clippel et al. (2014)) which, by allowing off-equilibrium set-valued outcomes, resolve the unfairness generated by dynamic mechanisms. To see the difference between our solution and the one based on dynamic veto mechanisms, consider a dynamic game that allows player 1 to veto $n+1-k$ alternatives and player 2 to veto $k-1$ of the remaining $k$ alternatives, where $k \in\{1, \ldots, n+1\} .{ }^{14}$ At each preference profile $\succ$, the subgame perfect equilibrium outcome of this game is the most preferred alternative of player 1 among $\mathrm{PV}_{v}(\succ)$ where $v_{1}=n+1-k$ and $v_{2}=k-1$. In other words, this dynamic veto mechanism subgame perfect implements a sub-correspondence of $\mathrm{PV}_{v}$ by refining it with respect to the true preference of the first mover. One could argue that fairness here could be achieved by selecting randomly the first-mover. Yet, this needs qualification since this randomization prevents some alternatives to arise as the following example shows. When $A=\{a, b, c, d, e\}$, at the preference profile $a \succ_{1} b \succ_{1} c \succ_{1} d \succ_{1} e$ and $c \succ_{2} b \succ_{2} a \succ_{2} d \succ_{2} e$, the dynamic veto mechanism which gives 2 vetoes to each voter implements, by alternating first movers, either $a$ or $c$ but excludes $b$. However, $\mathrm{PV}_{v}$ picks all three of $a, b$ and $c$. Thus, our simultaneous direct veto mechanisms allow for the implementation of the compromise alternative $b$ whereas their dynamic counterparts fail to do so. This constitutes a strong argument in favor of using simultaneous mechanisms.

We close by noting three limitations of our analysis. First, it is restricted to Nash implementation in pure strategies. Allowing for mixed strategies and exploring the existence of interesting DE mechanisms for settings with two or more players seems to be a promising research avenue (see Mezzetti and Renou (2012)). Second, the set of Nash-implementable SCRs expands if one considers implementation through non-DE mechanisms. Indeed, as long as BEB holds, the game-form associated to plurality rule Nash implements the union of tops ${ }^{15}$ which selects at each preference profile all alternatives that are top-ranked by at least one player. ${ }^{16}$ Third, we have considered implementation through ex-post Pareto efficient DE mechanisms. Other notions of efficiency are present in the literature such as stochastic dominance. Whether other SCRs can be Nash implemented through DE mechanisms by considering different notions of efficiency remains to be explored.

A possible extension of this work consists in exploring domain restrictions (such as singlepeakedness). If our possibility result obviously remains valid, it is possible that other mecha-

\footnotetext{
14 De Clippel et al. (2014) highlight that dynamic deterministic veto mechanisms (that they call shortlisting mechanisms) can also be helpful for implementation with two agents. In their mechanism, one agent starts the game by selecting $\frac{n+1}{2}$ alternatives and his opponent selects one alternative from the list. Two important differences exist between their approach and the current one: their implementation notion is dynamic and hence more permissive than the one used here (i.e. full Nash) and their focus is on deterministic mechanisms.

15 See Yeh (2008) for an axiomatization of this rule.

16 In this game form, each player announces a single alternative and one of them is selected randomly. Since it is a dominant strategy to announce one's best alternative, this mechanism is not $\mathbf{D E}$ as we may have two alternatives selected with positive probability in equilibrium.
} 
nisms arise as well, so that understanding the set of SCRs Nash-implementable through DEmechanisms on a restricted domain becomes a relevant question.

Likewise, with an infinite set of alternatives, the idea that players strike alternatives in such a way that some must remain can be implemented if the set of alternatives is a measurable space, has full measure 1 and players veto sets of measures $v_{1}$ and $v_{2}$, with $v_{1}+v_{2} \leq 1$ seems to be a relevant extension. Additional conditions must be imposed to make sure that some alternatives remain non-vetoed. These questions are another avenue of research that the present paper leaves open.

\section{Appendix A. Proof of Lemma 2}

We first state a result on the structure of the veto power that DE mechanisms generate.

Lemma A.1. Under the hypothesis of Theorem 2, for any partition $\{X, Y\}$ of $A$ with $X \in \mathcal{A} \backslash$ $\{\emptyset, A\}$, either $Y \in \operatorname{veto}(\mu, 1)$ or $X \in \operatorname{veto}(\mu, 2)$ but not both.

Proof. Let $\mu: \mathcal{M} \rightarrow \Delta$ be admissible and DE and let $X \in \mathcal{A} \backslash\{\emptyset, A\}$. Write $Y=A \backslash X$. Pick some $\succ \in \mathcal{L}_{A}^{2}$ such that $\forall x \in X, \forall y \in Y, x \succ_{1} y$ and $y \succ_{2} x$. Let $x^{*}$ and $y^{*}$ respectively denote the worst alternative for player 1 in $X$ and for player 2 in $Y: x \succ_{1} x^{*}$ for any $x \in X \backslash\left\{x^{*}\right\}$ and $y \succ_{2} y^{*}$ for any $y \in Y \backslash\left\{y^{*}\right\}$. The existence of such preference $\succ$ is ensured by our assumption that the domain contains all strict preferences on alternatives. Take also $\succeq^{*} \in \kappa(\succ)$ such that $p \succ_{1}^{*} q$ for all $p, q \in \mu(\mathcal{M})$ with $p[X]>0$ and $q[X]=0$, and such that $p \succ_{2}^{*} q$ for all $p, q \in \mu(\mathcal{M})$ with $p[Y]>0$ and $q[Y]=0$. The existence of such extended preference $\succeq^{*}$ is ensured by PREX; namely $\succeq_{1}^{*}$ stands for the PREX extension of $\succ_{1}$ for $x^{*}$ and $\succeq_{2}^{*}$ stands for the PREX extension of $\succ_{2}$ for $y^{*}$. Now suppose, for a contradiction, that $Y \notin \operatorname{veto}(\mu, 1)$ and $X \notin \operatorname{veto}(\mu, 2)$. Because $\mu$ is admissible and DE, there exists an equilibrium $m=\left(m_{1}, m_{2}\right) \in \mathcal{N}^{\mu}\left(\succeq^{*}\right)$ with $\mu(m)=\{a\}$ for some $a \in A$. Two cases are possible:

- Let $a \in X$. As $Y \notin \operatorname{veto}(\mu, 1), \exists m_{2}^{\prime} \in \mathcal{M}_{2}$ such that $\operatorname{supp}\left(\mu\left(m_{1}, m_{2}^{\prime}\right)\right) \cap Y \neq \varnothing$, hence $\mu\left(m_{1}, m_{2}^{\prime}\right) \succ_{2}^{*}\{a\}$ due to the construction of $\succ_{2}^{*}$, contradicting $m \in N^{\mu}\left(\succeq^{*}\right)$.

- Let $a \in Y$. As $X \notin \operatorname{veto}(\mu, 2), \exists m_{1}^{\prime} \in \mathcal{M}_{1}$ such that $\operatorname{SupP}\left(\mu\left(m_{1}^{\prime}, m_{2}\right)\right) \cap X \neq \varnothing$, hence $\mu\left(m_{1}^{\prime}, m_{2}\right) \succ_{1}^{*}\{a\}$, again due to the construction of $\succ_{1}^{*}$, contradicting $m \in \mathcal{N}^{\mu}\left(\succeq^{*}\right)$.

Thus, $Y \in \operatorname{veto}(\mu, 1)$ or $X \in \operatorname{veto}(\mu, 2)$. Because the mechanism is well-defined, it is impossible that a set belongs to veto $(\mu, 1)$ and its complement belongs to veto $(\mu, 2)$. Therefore either $Y \in$ $\operatorname{veto}(\mu, 1)$ or $X \in \operatorname{veto}(\mu, 2)$ but not both.

Proof of Lemma 2. Let $X \in \operatorname{veto}(\mu, 1), x \in X$ and $x^{\prime} \in A \backslash X .{ }^{17}$ Thus, there exists $m_{1} \in \mathcal{M}_{1}$ that vetoes $X$. The set $X^{\prime}=(X \backslash\{x\}) \cup\left\{x^{\prime}\right\}$ has the same cardinality as $X$. Write $Y=A \backslash(X \cup$ $\left.\left\{x^{\prime}\right\}\right)$, so that we have a partition

$$
A=(X \backslash\{x\}) \cup\{x\} \cup\left\{x^{\prime}\right\} \cup Y .
$$

Suppose, for a contradiction, that $X^{\prime} \notin$ veto $(\mu, 1)$. Lemma A.1 then implies that $Y \cup\{x\} \in$ veto $(\mu, 2)$. Therefore there exists $m_{2} \in \mathcal{M}_{2}$ that vetoes $Y \cup\{x\}$. Since $x^{\prime}$ is neither vetoed by $m_{1}$

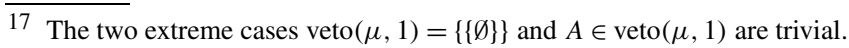


nor by $m_{2}, \mu\left(m_{1}, m_{2}\right)=\left\{x^{\prime}\right\}$. Now consider a unanimous preference profile $\succ=\left(\succ_{1}, \succ_{2}\right)$ such that $x \succ_{i} x^{\prime} \succ_{i} y$ for all $y \neq x, x^{\prime}$ and for $i=1,2$. For this preference profile, the second-best alternative $x^{\prime}$ is Pareto-dominated by $x$ but, at $\left(m_{1}, m_{2}\right)$, both players veto $x$. Thus, no unilateral deviation can obtain, with any probability, a better outcome than $x^{\prime}$. Thanks to BEB, that implies that $\left(m_{1}, m_{2}\right)$ is a Nash equilibrium, in contradiction with the Pareto efficiency assumption.

The proof of the proposition is established by noting that given any $X, X^{\prime} \in \mathcal{A} \backslash\{\varnothing, A\}$ with $\# X=\# X^{\prime}$, there is a finite sequence of sets $X=X_{1}, \ldots, X_{s}=X^{\prime}$ with $\#\left(X_{i} \cap X_{i+1}\right)=\# X-1$ for each $i \in\{1, \ldots, s-1\}$ and applying repeatedly the argument above.

\section{Appendix B. The role of PREX and Pareto efficiency in Theorem 2}

Example 1. Let $A=\{a, b, c, d\}$. For any player $i \in N$ and any strict preference $\succ_{i}$, only one extended preference is allowed: $\kappa\left(\succ_{i}\right)=\left\{\succ_{i}^{*}\right\}$, defined as follows: For any $p \in \Delta(A)$ with $\# \operatorname{SUPP}(p)>1, p \sim_{i}^{*} x$ where $x$ is the worst alternative in the support of $p$, that is the unique $x \in \operatorname{SUPP}(p)$ such that for all $y \in \operatorname{SUPP}(p), x \succeq_{i} y$. Notice that the domain $\kappa$ does not satisfy PREX but satisfies BEB.

Consider the mechanism $\mu$ in which each player can cast one or two vetoes. If both cast two vetoes, then the outcome is the uniform lottery on $A$, independently of what these vetoes are. If not, that is if at least one player casts only one veto, then the outcome is the uniform lottery over the set of non-vetoed alternatives. Formally, let $A^{[1]}$ and $A^{[2]}$ respectively denote the sets of one and two element subsets of $A$, then $\mathcal{M}_{1}=\mathcal{M}_{2}=A^{[1]} \cup A^{[2]}$. The outcome of the mechanism $\mu$ is a uniform lottery with support:

$$
\mu\left(m_{1}, m_{2}\right)=\left\{\begin{array}{cl}
A & \text { if } m_{1} \in A^{[2]} \text { and } m_{2} \in A^{[2]}, \\
A \backslash\left(m_{1} \cup m_{2}\right) & \text { otherwise. }
\end{array}\right.
$$

We now prove that, on the domain $\kappa$ this mechanism is admissible and DE in the domain $\kappa$. Moreover, $\mu$ Nash implements the $\operatorname{SCR~PV}_{(1,1)}=\mathrm{PV}_{(2,1)} \cup \mathrm{PV}_{(1,2)}$ that is not $\mathrm{PV}_{v}$ for $v_{1}+v_{2}=3$. This implies that any equilibrium outcome of $\mu$ is Pareto-efficient

The sub-game where player 1 plays in $A^{[1]}$ and player 2 plays in $A^{[2]}$ coincides with the one induced by the strike mechanism $\mu_{(1,2)}$ (that implements $\left.\mathrm{PV}_{(1,2)}\right)$. Consider an equilibrium of this sub-game: $\left(m_{1}, m_{2}\right) \in A^{[1]} \times A^{[2]}$. Player 1 has no profitable deviation in $A^{[1]}$, and a deviation to any strategy in $A^{[2]}$ yields the uniform lottery on $A$, which is the worst outcome and thus cannot be a profitable deviation. Player 2 has no profitable deviation in $A^{[2]}$; to see that player 2 has also no profitable deviation in $A^{[1]}$, write $A=\{a, b, c, d\}, m_{1}=\{a\}$ and $m_{2}=\{b, c\}$. Because $\mathrm{PV}_{(1,2)}$ is $\mathbf{D E}$, these alternatives are distinct and $\mu\left(m_{1}, m_{2}\right)=d$. A deviation of player 2 to $a$, $b$ or $c$ leaves $d$ in the support of the outcome, and is therefore not a profitable deviation. The deviation of player 2 to $d$ yields the outcome $\{b, c\}$. For the lottery on $\{b, c\}$ to be strictly better than $d$ for player 2, it must be that $d$ is ranked third or fourth in 2's preference. But this is not possible because we supposed $d$ is an equilibrium outcome in the veto mechanism where 2 has two vetoes. It follows that player 2 has no profitable deviation. We thus proved that the equilibria of the sub-game $A^{[1]} \times A^{[2]}$ are equilibria in the game-form associated to the mechanism $\mu$. Of course the same observation holds for the other sub-game $A^{[2]} \times A^{[1]}$. We will now show that there are no other equilibria.

If both players cast two vetoes $\mu\left(m_{1}, m_{2}\right)=A$ and one player deviating to vetoing (for instance) her worst alternative is a profitable response, so there is no equilibrium in the sub-game $A^{[2]} \times A^{[2]}$. If both players cast only one veto then the outcome is a lottery on two or three 
alternatives and a player can strictly improve by also vetoing his worst element among those non-vetoed by his opponent, so there is no equilibrium in the sub-game $A^{[1]} \times A^{[1]}$.

It follows that the mechanism $\mu$ is $\mathbf{D E}$ on this domain of extended preferences and implements the SCR PV $(1,1)$ which coincides with $\mathrm{PV}_{(2,1)} \cup \mathrm{PV}_{(1,2)}$.

Example 2. The following provides an example of a domain that satisfies BEB and PREX and there is a DE mechanism that is not Pareto efficient.

For this counter-example we consider the two-stage game such that player 1 at the first stage picks two and only two alternatives and player 2 chooses one of these at the second stage. The associated normal form game is as follows: Let $\mathcal{M}_{1}$ be the set $A^{[2]}$ of all two element subsets of $A$ and let $\mathcal{M}_{2}$ be the set of mappings $m_{2}: A^{[2]} \rightarrow A$ such that $\mu_{2}(x, y) \in\{x, y\}$. Define $\mu\left(m_{1}, m_{2}\right)$ as the (degenerated) lottery that assigns probability 1 to $\mu\left(m_{1}, m_{2}\right)=m_{2}\left(m_{1}\right) \in X$. Because the range of $\mu$ contains only degenerated lotteries, the properties BEB and PREX are trivially satisfied for any domain $\kappa$.

To prove that $\mu$ is admissible it is sufficient to note that the backward-induction equilibrium of the sequential game is a Nash equilibrium of $\mu$. Since all its outcomes are deterministic, $\mu$ is DE. To show that the SCR implemented by $\mu$ is not Pareto-efficient, we prove the existence of Pareto-dominated equilibrium. Let $A=\{a, b, c\}$ be the set of alternatives and let the two players agree on the ranking $a \succ b \succ c$. Let $m_{1}^{*}=\{b, c\}$ and let $m_{2}^{*}$ be the following:

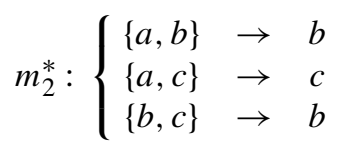

Then $\mu\left(m_{1}^{*}, m_{2}^{*}\right)=b$, which is Pareto dominated by $a$, and the reader will easily check that this is a Nash equilibrium. (Note that this equilibrium does not coincide with a subgame perfect equilibrium of the extensive form.)

Example 3. The following provides an example of a domain that satisfies BEB and PREX and there is a DE mechanism that Nash implements a SCR which is not Pareto efficient. The implemented SCR is not neutral.

Fix an alternative $a$. Each player can veto any subset of $A$. The outcome is a uniform lottery over non-vetoed alternatives and in case all alternatives are vetoed, the outcome is $a$. This mechanism is admissible: at every preference profile, both players vetoing all alternatives is an equilibrium that gives $a$ since no unilateral deviation alters the outcome. The mechanism is not deterministic but it is DE under BEB since whenever there is more than one alternative nonvetoed, it always a best response to veto the worst alternatives among the left non-vetoed by the opponent. It is not Pareto efficient since $a$ can be Pareto dominated and it is always an equilibrium outcome.

\section{Appendix C. Independence of $M M, I P, N$ and $P$}

We discuss in this section the independence of the four conditions, namely $M M, I P$, neutralon-its-vetoes $(N)$ and Pareto $(P)$ used to characterize Pareto-and-veto rules.

Lemma C.1. $N, P$ and $M M$ do not imply I $P$. 
Table 2

$f$ that satisfies $M M, P$ and $I P$ but fails $N$.

\begin{tabular}{lllllll}
\hline & $\begin{array}{l}a b c \\
(a b)\end{array}$ & $\begin{array}{l}a c b \\
(a c b)\end{array}$ & $\begin{array}{l}b a c \\
(b)\end{array}$ & $\begin{array}{l}b c a \\
(b)\end{array}$ & $\begin{array}{l}c a b \\
(c a b)\end{array}$ \\
\hline$a b c(a b)$ & $a$ & $a$ & $b$ & $b$ & $a$ & $b$ \\
$a c b \quad(a c b)$ & $a$ & $a$ & $b$ & $b$ & $b a, c\}$ & $c$ \\
$b a c \quad(b)$ & $b$ & $b$ & $b$ & $b$ & $b$ & $b$ \\
$b c a \quad(b)$ & $b$ & $b$ & $b$ & $b$ & $c$ & $c$ \\
$c a b \quad(c a b)$ & $a$ & $\{a, c\}$ & $b$ & $b$ & $c$ \\
$c b a$ & $(c b)$ & $b$ & $c$ & $b$ & $b$ & $c$ \\
\hline
\end{tabular}

Proof. Take $f=\mathrm{PV}_{v}$ with 0 vetoes and consider the profiles $\succ$ with $a \succ_{1} b \succ_{1} c$ and $b \succ_{2} c \succ_{2}$ $a$ and $\succ^{\prime}$ with $a \succ_{1}^{\prime} b \succ_{1}^{\prime} c$ and $b \succ_{2}^{\prime} a \succ_{2}^{\prime} c$. It follows that $f(\succ)=\{a, b, c\}$ and $f\left(\succ^{\prime}\right)=\{a, b\}$. Yet, $L\left(a, \succ_{2}\right)=a$ and $L\left(b, \succ_{1}^{\prime}\right)=\{b, c\}$, contradicting $I P$.

Lemma C.2. $N, M M, I P$ do not imply $P$.

Proof. Take $f$ that selects all alternatives not ranked last by some player. In the profile $\succ$ with $a \succ_{1} b \succ_{1} c$ and $a \succ_{2} b \succ_{2} c, f(\succ)=\{a, b\}$ while only $a$ is Pareto efficient.

Lemma C.3. $N, P, I P$ do not imply $M M$.

Proof. This is a direct consequence of Proposition 5.

Lemma C.4. $M M, P$ and I $P$ do not imply $N$.

Proof. Let $A=\{a, b, c\}$. Let $f$ be the SCR depicted in Table 2. In the table, the lines represent the preferences of player 1 and the columns the preferences of player 2. For short, $a b c$ stands for $a \succ_{i} b \succ_{i} c$ and so on. The rule $f$ is constructed as follows. For each $i$ if $\{a\},\{c\}$ or $\{a, c\}$ are ranked last for $i, a, c$ or both are eliminated. The non-vetoed alternatives are shown in the Table in parentheses next to the preferences. Then $f(\succ)$ contains all remaining Pareto efficient alternatives. The selected alternative(s) is indicated in the corresponding cell of the Table.

For instance $f(b a c, a c b)=\{b\}$ since player 1 has veto power over $\{a, c\}$ whereas 2 can't veto $\{b\}$. Similarly, $f(c a b, a c b)=\{a, c\}$ since $b$ is Pareto dominated by both $a$ and $c$ and no player has veto power over $\{b\}$, the common least preferred alternative.

This SCR is well-defined since a non-empty set is associated to each preference profile. By construction each player has veto power over $\{a\}$ and $\{c\}$ under $f$. For each player it maybe the case that $b$ is chosen when $b$ is her worst alternative; thus no player has veto power over $\{b\}$. Consequently $f$ does not satisfy $N$.

The rule $f$ satisfies $P$ by definition.

The condition $M M$ also holds since an alternative not going down in the voter's rankings is not harmed with the rule $f$. Indeed, if $x \in f(\succ)$, then $x$ is Pareto efficient and neither of the players can veto $\{x\}$ in $\succ$. For any $\succ^{\prime}$ with $\widetilde{L}\left(x, \succ_{i}\right) \subseteq \widetilde{L}\left(x, \succ_{i}^{\prime}\right) \forall i \in N, x$ remains Pareto efficient and neither of the players can veto $\{x\}$ in $\succ_{i}^{\prime}$, which implies that $x \in f\left(\succ^{\prime}\right)$ which implies that $M M$ holds.

We now prove that $f$ satisfies $I P$. Take $x \in f(\succ)$ and $y \in f\left(\succ^{\prime}\right)$. We wish to prove that $\widetilde{L}\left(x, \succ_{i}\right) \cap \widetilde{L}\left(y, \succ_{j}^{\prime}\right) \neq \emptyset$ and this is obvious if $x=y$; so let $x \neq y$. 
Consider first the case where neither $x$ nor $y$ equals $b$. Let, without loss of generality, $x=a$ and $y=c$. Take some player $i$. By definition of $f$, if $a=f(\succ)$, then $a$ is ranked last by no player at $\succ$, so $\widetilde{L}\left(a, \succ_{i}\right)$ contains some $z$ other than $a$. In case $a \succ_{i} c$, the condition for $I P$ is satisfied as $c=f\left(\succ^{\prime}\right)$ and $\widetilde{L}\left(c, \succ_{j}^{\prime}\right)$ contains $c$. In case $c \succ_{i} a, z$ must be $b$, so $\widetilde{L}\left(a, \succ_{i}\right)=\{a, b\}$. Again by definition of $f, \widetilde{L}\left(c, \succ_{j}^{\prime}\right)$ contains some $z$ other than $c$ and the condition for $I P$ holds whether $z$ is $a$ or $b$.

Consider second the remaining case where, without loss of generality, $x=b$ and $y \in\{a, c\}$. Say $y=a$ without loss of generality. Take player $i$. There are two subcases. In the first one $\widetilde{L}\left(b, \succ_{i}\right)=\{b\}$. Note that at $\succ_{j}^{\prime}, b$ cannot be ranked at top, as $j$ has veto power over $\{a, c\}$ which would contradict that $a \in f\left(\succ^{\prime}\right)$. If $\succ_{j}^{\prime}$ ranks $b$ the second best, then $a$ must be ranked top at $\succ_{j}^{\prime}$, as otherwise $a$ will be last in $\succ_{j}^{\prime}$ and hence $a \notin f\left(\succ^{\prime}\right)$ by the veto power of $j$ on $a$. Thus, $\widetilde{L}\left(a, \succ_{j}^{\prime}\right)$ contains $b$ which was in $\widetilde{L}\left(b, \succ_{i}\right)$, ensuring the $I P$ condition. Now consider the second subcase where $\widetilde{L}\left(b, \succ_{i}\right)$ contains some $z$ other than $b$. In case $z=a$, the condition for I $P$ is satisfied. Now let $z$ be $c$. So $a \succ_{i} b \succ_{i} c$. For the condition to fail, $a$ must be ranked last by $\succ_{j}^{\prime}$ which contradicts that $a \in f\left(\succ^{\prime}\right)$. We therefore conclude that $f$ satisfies $I P$.

\section{References}

Abreu, D., Sen, A., 1991. Virtual implementation in Nash equilibrium. Econometrica 59 (4), 997-1021.

Anbarci, N., 2006. Finite alternating-move arbitration schemes and the equal area solution. Theory Decis. 61 (1), 21-50.

Barberà, S., Coelho, D., 2010. On the rule of k-names. Games Econ. Behav. 70, 44-61.

Barberà, S., Coelho, D., 2019. On the Selection of Compromise Arbitrators. UAB. Mimeo.

Benoît, J.-P., Ok, E.A., 2008. Nash implementation without no-veto power. Games Econ. Behav. 64 (1), 51-67.

Bloom, D., Cavanagh, C., 1986. An analysis of the selection of arbitrators. Am. Econ. Rev. 76 (3), $408-422$.

Bochet, O., 2007. Nash implementation with lottery mechanisms. Soc. Choice Welf. 28 (1), 111-125.

Bochet, O., Maniquet, F., 2010. Virtual Nash implementation with admissible support. J. Math. Econ. 46 (1), $99-108$.

Bogomolnaia, A., Moulin, H., 2001. A new solution to the random assignment problem. J. Econ. Theory 100 (2), 295-328.

Busetto, J., Codognato, R., 2009. Reconsidering two-agent Nash implementation. Soc. Choice Welf. 32, 171-179.

De Clippel, G., Eliaz, K., Knight, B., 2014. On the selection of arbitrators. Am. Econ. Rev. 104, 3434-3458.

Dutta, B., 2019. Recent results on implementation with complete information. In: Social Design: Essays in Memory of Leonid Hurwicz. Springer.

Dutta, B., Sen, A., 1991. A necessary and sufficient condition for two-person Nash implementation. Rev. Econ. Stud. 58, 121-128.

Dutta, B., Sen, A., 2012. Nash implementation with partially honest individuals. Games Econ. Behav. 74, 154-169.

Ederer, F., Holden, R., Meyer, M., 2018. Gaming and strategic opacity in incentive provision. Rand J. Econ. 49 (4), 819-854.

Felsenthal, S.D., Machover, M., 1992. Sequential voting by veto: making the Mueller-Moulin algorithm more versatile. Theory Decis. 33 (3), 223-240.

Gärdenfors, P., 1976. Manipulation of social choice functions. J. Econ. Theory 13 (2), 217-228.

Herrero, M.J., Srivastava, S., 1992. Implementation via backward induction. J. Econ. Theory 56 (1), $70-88$.

Hurwicz, L., Schmeidler, D., 1978. Construction of outcome functions guaranteeing existence and Pareto optimality of Nash equilibria. Econometrica 46, 1447-1474.

Jackson, M.O., 2001. A crash course in implementation theory. Soc. Choice Welf. 18, 655-708.

Jackson, M.O., Sonnenschein, H.F., 2007. Overcoming incentive constraints by linking decisions. Econometrica 75 (1), 241-257.

Kelly, J.S., 1977. Strategy-proofness and social choice functions without singlevaluedness. Econometrica 45 (2), $439-446$.

Laslier, J.-F., Núñez, M., Pimienta, C., 2017. Reaching consensus through approval bargaining. Games Econ. Behav. 104, 241-251.

Maskin, E., 1999. Nash equilibrium and welfare optimality. Rev. Econ. Stud. 66, 23-38.

Matsushima, H., 1988. A new approach to the implementation problem. J. Econ. Theory 45 (1), 128-144. 
Mezzetti, C., Renou, L., 2012. Implementation in mixed Nash equilibrium. J. Econ. Theory 147 (6), $2357-2375$.

Moore, J., Repullo, R., 1988. Subgame perfect implementation. Econometrica 96 (5), 1191-1220.

Moore, J., Repullo, R., 1990. Nash implementation: a full characterization. Econometrica 58, 1083-1099.

Moulin, H., 1981. Prudence versus sophistication in voting strategy. J. Econ. Theory 24 (3), 398-412.

Moulin, H., 1983. The Strategy of Social Choice. Advanced Textbooks in Economics. North-Holland.

Mueller, D.C., 1978. Voting by veto. J. Public Econ. 10 (1), 57-75.

Núñez, M., Laslier, J.-F., 2015. Bargaining through approval. J. Math. Econ. 60, 63-73.

Sanver, M.R., 2006. Nash implementing non-monotonic social choice rules by awards. Econ. Theory 28 (2), $453-460$.

Sanver, M.R., 2018. Implementing Pareto optimal and individually rational outcomes by veto. Group Decis. Negot. 27 (2), 223-233.

Yeh, C.-H., 2008. An efficiency characterization of plurality rule in collective choice problems. Econ. Theory 34 (3), $575-583$. 